\title{
Conserved charges in (Lovelock) gravity in first order formalism
}

\author{
Elias Gravani:* \\ (Dated: March 3, 2018)
}

\begin{abstract}
We derive conserved charges as quasi-local Hamiltonians by covariant phase space methods for a class of geometric Lagrangians that can be written in terms of the spin connection, the vielbein and possibly other tensorial form fields, allowing also for non-zero torsion. We then re-calculate certain known results and derive some new ones in three to six dimensions hopefully enlightening certain aspects of all of them. The quasi-local energy is defined in terms of the metric and not its first derivatives, requiring 'regularization' for convergence in most cases. Counter-terms consistent with Dirichlet boundary conditions in first order formalism are shown to be an efficient way to remove divergencies and derive the values of conserved charges, the clear-cut application being metrics with AdS (or dS) asymptotics. The emerging scheme is: all is required to remove the divergencies of a Lovelock gravity is a boundary Lovelock gravity.
\end{abstract}

\section{INTRODUCTION}

Defining energy in a consistent and general manner is exceedingly difficult in general relativity. One good reason is that unlike any other field theory it is hard to localize energy in gravity: its density should be reasonably constructed out of first derivatives of the metric which vanish at any point for inertial observers. Formally, energy is the generator of a local symmetry thus it should vanish locally. Another reason of similar nature, is that energy, by shaping spacetime itself, is not defined in some absolute manner: it depends strongly on boundary conditions, which introduce the asymptotics i.e the class of metrics one intends to study. Implicitly or explicitly the conditions define a reference background, for which energy is zero.

In general relativity, the Bondi-Sachs mass 1] [2] defined in the null infinity and the ADM mass [3] defined in the spatial infinity, are accepted definitions of energy for asymptotically flat spacetimes. ADM mass is interpreted as the total energy of spacetime. Abbott-Deser mass [4] provides a definition for asymptotically de Sitter spacetimes, in the spirit of ADM mass. All these definitions are written as an integral over a co-dimension two spatial sphere. This is an implication of non-localizability.

Quasi-localization is a most interesting alternative. The word means: we consider the total energy content of finite spatial regions, of size (and shape) otherwise arbitrary. Of all definitions, some of which are mentioned in section II C. the Brown-York quasi-local energy [5] is especially appealing. By the generalization of 6 [7], which originated from the 'AdS /CFT correspondence' [8] [9], the definition applies to spacetimes with $A d S$ or even de Sitter asymptotics in a natural manner. Moreover, both the Brown-York definition and the famous correspondence are in the spirit of 'holography' 10] 11]: the gravitational degrees of freedom are fundamentally boundary degrees of freedom.

Even if one is not particularly interested in the localization problem, the Brown-York quasi-local energy, as a means to define energy in spacetime, is technically attrac-

*Electronic address: eliasgravanis@netscape.net tive. It derives from the invariant action functional as a generator in the sense of Hamilton-Jacobi theory [5] 12]. Such a definition is still applicable when one departs from general relativity.

It has become customary in recent years to consider curved spacetimes of dimension higher than four. Once such a choice is made the elementary conditions that uniquely determine the Einstein equations in four dimensions are not adequate. In dimension five or higher there exist rank two, symmetric and covariantly conserved tensors involving up to second derivatives of the metric tensor. These are the Lovelock tensors [13]. Generic linear combinations of them define equally good field equations for the metric tensor. Moveover the field equations are second order differential equations, giving 'Lovelock gravity' a feeling of familiar ground. The Cauchy problem appears almost- though not entirely- analogous to that in general relativity [14].

The action functional for Lovelock gravity is very interesting (starting from the Einstein-Hilbert action itself). Written in tensor notation, each Lovelock Lagrangian has the form of a topological density of some lower (even) dimension, only now its indices are summed over a higher number of dimensions. This dimensional translation provides local dynamics for the metric tensor: Einstein and general Lovelock field equations.

This is mysteriously neat. The mystery is partly dissolved if we, as Zumino did [15], get more geometric. For our needs useful mathematical references are [16] 17]. The metric tensor may be replaced by the vielbein $E^{a}$ i.e. a basis in an abstract Lorentz vector bundle with metric $\eta_{a b}$ and volume form $\epsilon_{a \cdots b}$. The spacetime metric tensor reads $g=\eta_{a b} E^{a} \otimes E^{b}$. A connection $\omega_{b}^{a}$ on the Lorentz bundle may or may not be Levi-Civita, and let $\Omega_{b}^{a}:=d \omega^{a}{ }_{b}+\omega^{a}{ }_{c} \omega^{c}{ }_{b}$ denote its curvature. (The exterior product is understood). The general Lovelock Lagrangian is constructed by the Lorentz invariant form $\epsilon(\Omega \cdots \Omega E \cdots E) \equiv \epsilon_{a b \cdots c d e \cdots f} \Omega^{a b} \cdots \Omega^{c d} E^{e} \cdots E^{f}$. We integrate this density over a manifold without boundary, to get an action. The difference between Einstein and Lovelock gravity is that the latter contains terms with more than one $\Omega$ factors. First order formalism means treating $E$ and $\omega$ as independent variables. 
Varying w.r.t. $\omega$ the curvature changes by $\delta \Omega=D(\delta \omega)$, where $D$ is the covariant derivative. The curvature satisfies $D \Omega=0$, the Bianchi identity. Varying the action w.r.t. to $\omega$ we get, after an integration by parts, terms involving $D E$. If there no $E$ factors in the Lagrangian the variation vanishes identically: it is a topological density. The torsion, $D E$, vanishes iff the connection is Levi-Civita. Upon restricting ourselves to such connections the variation vanishes identically (in Einstein gravity we have no choice); then the Lovelock tensors and field equations are obtained, purely algebraically, by varying the action w.r.t. $E$.

Technically there is a bonus in the first order formulation. Once one is free to allow for torsion, one may consider a generalization of Lovelock gravity such that connection is not Levi-Civita, as well as consider other Lorentz invariant densities built by contraction with the invariant tensors $\delta_{[b}^{a} \cdots \delta_{c]}^{d}$. These are related to the Pontryagin invariants as opposed to Lovelock terms which are related to the Euler number. These Lorentz invariants will be our toy model of a geometric gravitational Lagrangian. Most of our examples though will be in Lovelock gravity.

In section IIA the covariant phase space methods as applied to a gravitational action are discussed. In section we derive the Hamiltonian in this framework based on the Poincare invariance of the symplectic form, and distinction between cases where this can and cannot be done is made, type and (I) and (II) cases respectively. In section $\amalg \mathbf{C}$ the quasi-local value of the charges is introduced. In section IIE the type (II) case Hamiltonian is derived. Between sections [II-V various results in Einstein gravity including torsion are collected and discussed. Matters such as a possibility for attributing a non-zero mass to Minkowski space in certain gravity theories is analyzed. In section VI an example of Lovelock gravity, the case of five dimensions, is discussed in detail. In section VII a brief excursion in dimension six shows that the Dirichlet boundary forms are adequate to remove the divergencies and obtain results obtained by other methods. It is also understood that there is a general scheme: at least in the first order formalism, all is required to remove the divergencies of a Lovelock gravity is a boundary Lovelock gravity. In section VIII the quasi-local mass is used to interpolate between $A d S$ and flat spacetime results. A brief discussion on boundary forms is in the appendix.

As a matter of terminology, one should distinguish the 'canonical generator' in the sense of Hamiltonian theory which involves Poisson brackets, from that in the sense of Hamilton-Jacobi theory where generators are defined as derivatives of Hamilton's Principal Function that is the action functional on-shell. Of course, the two kinds are intimately related; the covariant phase space framework provides the link. But there are differences. One obtains respectively: the value of the total (over all space) value of the charge, and the actual quasi-local value over a finite spatial region. But we shall work everything out in a quasilocal setting: the boundary Lagrangian will be of crucial importance. Thus everything is quasi-local and what is what will be understood from context. After all, in the co- variant phase formulation one can easily see that the family of functions (with radius as parameter) whose limit is the Hamiltonian generator are the Hamilton-Jacobi generators, section IC. Having said that we shall not be very careful about terminology for the rest of this paper.

\section{THE CONSERVED CHARGE AS CANONICAL GENERATOR}

\section{A. Action and invariant symplectic form}

Let a region $\mathcal{R}$ be an arbitrary open set spacetime $M$. Let $\Psi^{A \cdots}$ be a collection of vector valued forms over $M$. (The Lorentz indices of the bulk fields are now denoted by capital letters.)Let fields intrinsic to the boundary $\partial \mathcal{R}$ of the region $\mathcal{R}$ be denoted by $\Psi_{\|}^{a \ldots}$. We will use this same symbol $\Psi_{\|}$for bulk fields which agree with the intrinsic ones when pulled back into $\mathcal{R}$.

The induced fields into the boundary are $i_{\|}^{*} \Psi^{a \cdots}$. In every smooth component of the boundary $\partial \mathcal{R}$, and as long as these components are 'orthogonal' [12], the intrinsic fields can be chosen to coincide with the induced values of the bulk fields: $\Psi_{\|}^{a \ldots}=i_{\|}^{*} \Psi^{a \cdots}$. We shall be content with the 'orthogonal' case.

Consider a local functional of the fields

$$
S=\int_{\mathcal{R}} \mathcal{L}+\int_{\partial \mathcal{R}} i_{\|}^{*} \mathcal{B}_{(C)},
$$

where $\mathcal{L}$ is a functional of the fields $\Psi$, the Lagrangian of the theory. $\mathcal{B}_{(C)}$ is a form in $\mathcal{R}$ that depends on $\Psi$ and $\Psi_{\|}$and it is such that: Under given conditions $C$ for the variations $\delta_{(C)}$ of the fields $\Psi$ and $\Psi_{\|}$, the action functional $S$ has an extremum

$$
\delta_{(C)} S=0,
$$

when the Euler-Lagrange equations are satisfied by $\Psi$ in $\mathcal{R}$.

We want first to find and analyze a formula for the total energy and angular momentum, derived as canonical generators conjugate to the associated diffeomorphisms, for the theory of fields $\Psi$ given by the Lagrangian $\mathcal{L}$. Our variables $\Psi$ will be the vielbein $E$ and the Lorentz connection $\omega$, as well as other 'matter' fields which we shall not explicitly mention. Our toy model, and quite the objective, will be Lagrangian $\mathcal{L}$ which is a linear combination of local Lorentz invariants of the curvature $\Omega$ and $E$. There can also be 'matter' Lagrangian terms which will be left understood.

As a warmup, consider an action in particle mechanics: $S=\int_{t_{1}}^{t_{2}} d t L(q, \dot{q})$. Let's define the phase space of the theory to mean the space of solutions of the classical equations [18], denoted compactly as $\mathcal{E}=0$. On the phase space of the histories $q$ infinitesimal variations $\delta q$ are tangent directions on that space and can be thought of as vector fields. They are solutions to the linearized equations of motion $\delta \mathcal{E}=0$. It is quite convenient to define 
an exterior derivative $\boldsymbol{\delta}$ over the phase space. Its nilpotence, $\boldsymbol{\delta}^{2}=0$, acting on the fields means that: We choose a multi-parameter family of fields twice continuously differentiable w.r.t. these parameters, variation means differentiation w.r.t. a parameter and the order of differentiations is irrelevant. We have:

$$
\boldsymbol{\delta}^{2} S=[\boldsymbol{\delta} p \boldsymbol{\delta} q]_{t_{1}}^{t_{2}}-\int_{t_{1}}^{t_{2}} d t \boldsymbol{\delta} q \cdot \boldsymbol{\delta} \mathcal{E}
$$

where of course $p=\partial L / \partial \dot{q}$. By the nilpotence of $\boldsymbol{\delta}$ we have that $\boldsymbol{\delta}^{2} S=0$. So for on-shell variations we get $[\boldsymbol{\delta} p \boldsymbol{\delta} q]_{t_{1}}^{t_{2}}=0$ i.e. $\boldsymbol{\delta} p \boldsymbol{\delta} q$ is unchanged through time.

The importance of the phase space 2 -form $\boldsymbol{\sigma}:=\boldsymbol{\delta} p \boldsymbol{\delta} q$ is that it gives the clue to formulate the Hamiltonian theory in a relativistically covariant way [18]. Phase space has here explicit coordinates $x=\left(x^{1}, x^{2}\right)=(p, q)$. The form $\boldsymbol{\sigma}$ is closed $\boldsymbol{\delta} \boldsymbol{\sigma}=0$ and non-degenerate i.e. its components $\sigma_{12}=-\sigma_{21}=1$ form an invertible matrix. Now if on a phase space we can find an closed and non-degenerate twoform we say we have a symplectic structure. The crucial theorem of Darboux [19] says that we can always find coordinates such that locally a symplectic structure can be written in the form $\boldsymbol{\delta} p \boldsymbol{\delta} q$. Therefore we have the essential structure to do canonical theory without any unnessecary choice of coordinates that breaks covariance. If the symplectic structure does not depend on time, as above, then it is a Poincare invariant. Thus one succeeds in formulating a covariant canonical theory. Hamilton's equations can be written as $\dot{x}^{i} \boldsymbol{\sigma}_{i j}=-\partial_{j} H$. Phase space components need not necessarily appear as we will see below. The symplectic methods in Hamiltonian and Lagrangian mechanics are discussed in depth in [20] and [19].

Let's turn now to the theory of fields $\Psi$ and our toy model. We have

$$
\delta S=\int_{\partial \mathcal{R}} \delta \omega \cdot \frac{\partial \mathcal{L}}{\partial \Omega}+\delta \mathcal{B}_{(C)}+\int_{\mathcal{R}} \delta \Psi \cdot \mathcal{E}
$$

$\boldsymbol{\delta}$ is thought of as anti-commuting with the exterior derivative $d, \boldsymbol{\delta} d+d \boldsymbol{\delta}=0$, and the same with all $p$-forms with $p$ odd.

Taking the second variation we have:

$$
0=\delta^{2} S=\int_{\partial \mathcal{R}} \delta\left\{\delta \omega \cdot \frac{\partial \mathcal{L}}{\partial \Omega}+\delta \mathcal{B}_{(C)}\right\}+\int_{\mathcal{R}} \delta \Psi \cdot \delta \mathcal{E} .
$$

The variations $\delta \Psi$ are solutions to the linearized equations of motion $\delta \mathcal{E}=0$.

For a field theory over space and time the boundary $\partial \mathcal{R}$ is closed hypersurface in spacetime. Also an instant of time means a spacelike hypersurface. Let two hypersurfaces $\Sigma_{1}$ and $\Sigma_{2}$ and let $\mathcal{R}$ be the region between them. The boundary of $\mathcal{R}$ is closing with a timelike hypersurface at spatial infinity. To bring that a little closer let two spacelike hypersurfaces $\Sigma_{1}^{\alpha}$ and $\Sigma_{2}^{\alpha}$ whose boundaries $S_{1}^{\alpha}$ and $S_{2}^{\alpha}$ are connected by a (mostly) timelike hypersurface $T^{\alpha}$ such that as $\alpha \rightarrow \infty$ the boundaries $S^{\alpha}$ approach spheres at spatial infinity.
In order to obtain a Poincare invariant symplectic structure in field theory we need a 'no-leaking' condition: The part of the boundary integral over $T^{\alpha}$ in (5) goes to zero as $\alpha \rightarrow \infty$ :

$$
i_{T^{\alpha}}^{*}\left(\boldsymbol{\delta}\left\{\boldsymbol{\delta} \omega \cdot \frac{\partial \mathcal{L}}{\partial \Omega}+\delta \mathcal{B}_{(C)}\right\}\right) \rightarrow 0,
$$

as $\alpha \rightarrow \infty$. To proceed from this point one should determine and define carefully the asymptotic symmetries at spatial infinity, define the space of fields whose asymptotics preserve these symmetries, and make sure that they are such that the quantity above vanishes in the limit. This is already quite involved in Einstein gravity and will not try to be rigorous; to obtain a result we have to proceed somehow else. We shall assume that there are asymptotic conditions on the fields such that the 'no-leaking' condition (6) holds and see what do we get from that.

Condition (6) implies that locally in phase space

$$
i_{T^{\alpha}}^{*}\left(\boldsymbol{\delta} \omega \cdot \frac{\partial \mathcal{L}}{\partial \Omega}+\boldsymbol{\delta} \mathcal{B}_{(C)}\right) \rightarrow i_{T^{\alpha}}^{*} \boldsymbol{\delta} B_{\infty},
$$

in the limit $\alpha \rightarrow \infty$, for some phase space function $B_{\infty}$. Define a 'regularized' boundary form: $\mathcal{B}_{(C)}^{\text {reg }}:=\mathcal{B}_{(C)}-B_{\infty}$. Then

$$
i_{T^{\alpha}}^{*}\left(\boldsymbol{\delta} \omega \cdot \frac{\partial \mathcal{L}}{\partial \Omega}+\delta \mathcal{B}_{(C)}^{r e g}\right) \rightarrow 0
$$

as $\alpha \rightarrow \infty$. Upon replacing $\mathcal{B}_{(C)}$ with this boundary form from the level of the Lagrangian (1), we work with a boundary form such that the 'no-leaking' conditions holds in the form (8).

Before proceeding let us digress briefly for some enlightening comments. First of all let's return to equation (4). If we restrict ourselves to variations $\boldsymbol{\delta}=\boldsymbol{\delta}_{(C)}$ which respect the conditions $C$ in the given region, by the very definition of the boundary form we have

$$
i_{\|}^{*}\left(\boldsymbol{\delta}_{(C)} \omega \cdot \frac{\partial \mathcal{L}}{\partial \Omega}+\boldsymbol{\delta}_{(C)} \mathcal{B}_{(C)}\right)=0
$$

and $S$ attains an extremum for $\mathcal{E}=0$ in the interior of the region.

Dirichlet boundary conditions amount to consider variations such that the induced fields are held fixed. That means that $\mathcal{B}_{(C)}$ is defined within a class of boundary forms which satisfy (9) up an arbitrary functional of the induced fields. The idea is that one might be able to find within that class a boundary form, denoted by $\mathcal{B}_{(C)}^{\text {reg }}$, such that the stronger 'no-leaking' condition (8).

Other boundary conditions such that a boundary form satisfying both (9) and (8) are also possible. They are discussed in section IID,

If $\boldsymbol{\delta}_{(C)}$ are replaced with general variations the r.h.s. of (9) does not vanish. Instead defines the 'quasi-local' energy-momentum and spin tensors $\Theta_{\text {quasi-local: }}$

$$
\boldsymbol{\delta} \omega \cdot \frac{\partial \mathcal{L}}{\partial \Omega}+\delta \mathcal{B}_{(C)}^{\text {reg }}=\boldsymbol{\delta} \Psi \cdot \Theta_{\text {quasi-local }},
$$


where the pullback into the boundary is understood. The terminology comes from the fact that for Dirichlet boundary conditions $\Theta_{\text {quasi-local }}$ is indeed the quasi-local energy tensors of Brown and York [5]. The modification to the 'regularized' boundary form originated from the $A d S / C F T$ correspondence [8] whose framework suggests that local boundary counter-terms should cure divergencies 9]. It was then applied successfully to $A d S$ mass calculations in [6] following also Ref. 21]. (It was also shown that by the same formal methods one can obtain results for de Sitter spacetimes 7] though there are minus signs ambiguities due to nature of $d S$ space [22] [23]).

The point is that if the quasi-local tensors are finite then indeed the r.h.s. of (10) goes to zero at infinity, as $\delta \Psi$ vanishes in that limit for any definition of the function space of the fields. In other words the 'no-leaking' condition (8) is satisfied. In other words the symplectic form is Poincare invariant.

The basic problem then is whether we know how to construct the form $B_{(C)}^{r e g}$ such that (8) holds. It will be quite useful in stating certain simple observations in this work to distinguish between the following three cases.

Type (I). We know how to construct $B_{(C)}^{r e g}$. Then the conserved charges quasi-local in nature. Everything depends on the finiteness of the quasi-local tensor. This guaranties that (7) can be satisfied within a reasonable field space i.e. the asymptotics of the field $\Psi$ will allow for variations of the parameters of the solutions in $\delta \Psi$. Stronger conditions on asymptotics would invalidate the whole analysis. We are clearly very heuristic here.

Type (II). We do not know how to write down a simple enough $B_{(C)}^{r e g}$. This is especially the case of asymptotically flat spacetimes.

Of course, the 'we know' and 'we do not know' part in the above rather sketchy definitions depends on assumptions. For example, for the asymptotically flat case, fairly complicated boundary terms have been presented in works such as 24 27] which do produce convergent results. These are related to works such as [28] where another boundary term is constructed by a 'light-cone reference'; this term produces a quasi-local mass with nice properties in the 'small sphere limit'. We will not attempt to add something new on these matters so we have nothing to offer in those directions.

We would like, among other things, to obtain values for the conserved charges by simple prescriptions in a uniform way in the various dimensions and theories (of interest). The boundary forms $B_{(C)}^{r e g}$ shall be the simplest polynomials of forms constructed out of the vielbein and connections one-forms their derivatives. This is adequate in many cases, as far as the values for the charges are concerned. This is particularly useful in Lovelock gravity where formulas can get a lot messier than in Einstein gravity. The use of differential forms notation throughout is also a choice made in that spirit.

Type (H) ('H' stands for 'Hybrid'). This is a special case of (I) such that no 'corrections' are required: a minimal boundary form consistent with the boundary conditions is adequate for convergence.
The following definition will be completely clear after the formulas have been derived.

Definition II.1. The first two cases correspond to two different derivations of the Hamiltonian generators. If a Hamiltonian is calculated as type (I) it will be called a Brown-York Hamiltonian. If it is calculated as a type (II) it will be called a relative Hamiltonian generator. As the latter is defined through weaker conditions a Brown-York generator can always be thought of as a relative generator; one may have only fix a $\boldsymbol{\delta}$-integration constant appropriately. The hybrid case type $(\mathbf{H})$ is one such that a relative generator is also a Brown-York generator.

In what follows the derivations are not essentially different in spirit from the covariant phase space methods of Wald and collaborators 29 32] though the style is closer to that of Ref. [18]. The difference lies in we work throughout with first order formalism which allows us to obtain the results in a closed form for the Lagrangians under consideration, and in that we take the existence of a well defined Poincare invariant symplectic form as the guiding principle of the derivations.

\section{B. The Hamiltonian generator in type (I)}

Suppose we know how to construct explicitly a boundary form $\mathcal{B}_{(C)}$ such that (8) holds.

Note II.2. From now on we drop the index 'reg' for a boundary form satisfying (8).

We have a candidate for a symplectic form:

$$
\boldsymbol{\sigma}:=\boldsymbol{\delta} \int_{\Sigma}\left\{\boldsymbol{\delta} \omega \cdot \frac{\partial \mathcal{L}}{\partial \Omega}+\delta \mathcal{B}_{(C)}\right\}
$$

i.e. one that is independent of the spacelike hypersurface $\Sigma$. Finiteness is also required but will be left as an assumption. It will be rather clear form the very conditions imposed.

$\sigma$ is a phase space 2 -form which is by construction closed under the exterior derivative: $\delta \boldsymbol{\sigma}=0 . \boldsymbol{\sigma}$ it is not a symplectic form because it is degenerate: it vanishes when $\delta \Psi$ are gauge transformations. We see below that $\boldsymbol{\sigma}$ will naturally project into a subspace normal to the gauge transformation directions.

Let $\xi$ be a vector field over the spacetime manifold $M$. Then $£_{\xi} \Psi$ can be regarded as a tangent vector over the phase space. Let a phase space function $H_{\xi}$ such that

$$
\boldsymbol{i}_{£_{\xi} \Psi} \boldsymbol{\sigma}=-\boldsymbol{\delta} H_{\xi} .
$$

$\left(\boldsymbol{i}_{\Xi}\right.$ is the inner product on forms in phase space with the phase space vector $\boldsymbol{\Xi}$.) $H_{\xi}$ is the Hamiltonian generator in phase space, or simply the Hamiltonian, of the transformations generated by $\xi$ in spacetime. Relations (12) are Hamilton's equations.

Here we got an action and a symplectic form $\boldsymbol{\sigma}$ but not a Hamiltonian. The transformations generated by $\xi$, the diffeomorphisms, are a symmetry of the action and therefore 
of $\boldsymbol{\sigma}$. The Hamiltonian can be constructed through the existence of the symmetry as a momentum mapping [20] essentially using a Noether type method.

If $\boldsymbol{\sigma}$ is invariant along a vector $\boldsymbol{\Xi}$ in phase space this formally means that $£_{\Xi} \boldsymbol{\sigma}=0$. By the closure of $\boldsymbol{\sigma}$ this means that $\boldsymbol{\delta}\left(\boldsymbol{i}_{\Xi} \boldsymbol{\sigma}\right)=0$. We used Cartan's relation for the Lie derivative in phase space: $£_{\Xi}=\boldsymbol{\delta} \boldsymbol{i}_{\Xi}+\boldsymbol{i}_{\Xi} \boldsymbol{\delta}$. Thus the phase space one-form $\boldsymbol{i}_{\Xi} \boldsymbol{\sigma}$ must be exact: $\boldsymbol{i}_{\Xi} \boldsymbol{\sigma}=-\boldsymbol{\delta} H$, for some phase space function $H$. That this holds globally is an assumption. Now if the 'Hamiltonian' $H$ is given, and the $\boldsymbol{\sigma}$ is non-degenerate, then the last relation defines a Hamiltonian vector field $\boldsymbol{\Xi}_{H}$. Hamilton's equations hold along its integral curves $£_{\xi} \Psi=\boldsymbol{\Xi}_{H}$.

Now $\boldsymbol{\sigma}$ being diffeomorphism invariant means that it does change along the directions on the tangent of the phase space that correspond to diffeomorphism transformations of the fields. That is $£_{£_{\xi} \Psi} \boldsymbol{\sigma}=0$. Through a reasoning similar to the above, we arrive at (12) for some phase space function $H_{\xi}$ which we must construct, the Hamiltonian generators of transformations over $M$ generated by $\xi$.

A debt left from earlier is that $\boldsymbol{\sigma}$ does project on the space of solutions modulo gauge transformations, by having no components in those directions. This statement translates to: $\boldsymbol{i}_{£_{\xi} \Psi} \boldsymbol{\sigma}=0$. This is the case when $\xi$ has a compact support in $M$, or better corresponds to proper gauge transformations in the language of 33 34. Then $H_{\xi}$ like all gauge symmetry generators vanish identically on-shell, as generators of local symmetries should do, and $\sigma$ projects. When $\xi$ are non-trivial asymptotically, are improper diffeomorphisms, the generators become non-trivial tensorial quantities of the asymptotic symmetry groups. They should of course be related to the asymptotic values of the fields.

Regge-Teitelboim [33] produce the generators starting with the Hamiltonian as the usual Legendre transform of the Lagrangian, and postulating additional suitable boundary integrals which make all the required functional derivatives well defined. There is quite a bit of analogy between that construction and covariant phase space methods which work with action functional, only in the latter the canonical variables are not explicitly picked. The final on-shell must agree wherever the methods make sense though the results may differ by phase space constants i.e. parameters that depend on the theory (couplings) and not the solution. This happens explicitly in the case of locally flat spacetime in three dimensions, as well shall see.

Finally, without dwelling further into the symplectic form of the Hamiltonian theory we will find an $H_{\xi}$ starting from (12) and (11). We have

$$
\boldsymbol{\sigma}=\int_{\Sigma} \boldsymbol{\delta} \omega \cdot \boldsymbol{\delta} \frac{\partial \mathcal{L}}{\partial \Omega}
$$

We have

$$
\boldsymbol{i}_{£_{\xi} \Psi} \boldsymbol{\sigma}=\int_{\Sigma} £_{\xi} \omega \cdot \boldsymbol{\delta} \frac{\partial \mathcal{L}}{\partial \Omega}+\boldsymbol{\delta} \omega \cdot £_{\xi} \frac{\partial \mathcal{L}}{\partial \Omega} .
$$

By $\delta £_{\xi}=£_{\xi} \boldsymbol{\delta}$ the integrant can also be written as

$$
-\delta\left\{£_{\xi} \omega \cdot \frac{\partial \mathcal{L}}{\partial \Omega}\right\}+£_{\xi}\left\{\delta \omega \cdot \frac{\partial \mathcal{L}}{\partial \Omega}\right\} .
$$

Using $\boldsymbol{\delta} \Omega=-D \boldsymbol{\delta} \omega$ and $£_{\xi} \omega=D\left(i_{\xi} \omega\right)+i_{\xi} \Omega$ one can easily show that the first bracket reads

$$
d\left(i_{\xi} \omega \cdot \frac{\partial \mathcal{L}}{\partial \Omega}\right)+i_{\xi} \mathcal{L}-i_{\xi} \Psi \cdot \mathcal{E} .
$$

This is a quite useful fact. Then it is easy to show that the whole of (15) reads

$$
\begin{aligned}
& -\delta d\left(i_{\xi} \omega \cdot \frac{\partial \mathcal{L}}{\partial \Omega}\right)+d i_{\xi}\left(\delta \omega \cdot \frac{\partial \mathcal{L}}{\partial \Omega}\right) \\
& +\delta \Psi \cdot i_{\xi} \mathcal{E}+i_{\xi} \Psi \cdot \delta \mathcal{E}
\end{aligned}
$$

$\boldsymbol{\delta} i_{\xi}=-i_{\xi} \boldsymbol{\delta}$ is used often. We have kept the terms involving $\mathcal{E}$ and $\delta \mathcal{E}$ in this formula for completeness. So we have that $\boldsymbol{i}_{£_{\xi} \Psi} \boldsymbol{\sigma}$ equals

$$
-\delta \int_{S^{\alpha}} i_{\xi} \omega \cdot \frac{\partial \mathcal{L}}{\partial \Omega}+\int_{S^{\alpha}} i_{\xi}\left(\delta \omega \cdot \frac{\partial \mathcal{L}}{\partial \Omega}\right),
$$

where the limit $\alpha \rightarrow \infty$ is understood.

Now $S^{\alpha}$ is a boundary of both $\Sigma^{\alpha}$ and $T^{\alpha}$. We may orient the manifold $T^{\alpha}$ to be such that $\xi$ is tangent to it at the boundary $S^{\alpha}$. Then the 'no-leaking' condition (8), extended to the boundary of $T^{\alpha}$, tells us that

$$
i_{S^{\alpha}}^{*} i_{\xi}\left(\delta \omega \cdot \frac{\partial \mathcal{L}}{\partial \Omega}\right) \rightarrow-i_{S^{\alpha}}^{*} i_{\xi} \boldsymbol{\delta} \mathcal{B}_{(C)},
$$

as $\alpha \rightarrow \infty$. Thus we have

$$
\boldsymbol{i}_{£_{\xi} \Psi} \boldsymbol{\sigma}=-\boldsymbol{\delta}\left\{\int_{S^{\alpha}} i_{\xi} \omega \cdot \frac{\partial \mathcal{L}}{\partial \Omega}+i_{\xi} \mathcal{B}_{(C)}\right\} .
$$

By (12) we have

$$
H_{\xi}=\int_{S^{\infty}}\left\{i_{\xi} \omega \cdot \frac{\partial \mathcal{L}}{\partial \Omega}+i_{\xi} \mathcal{B}_{(C)}\right\}+H_{\xi}^{0} .
$$

(12) defines the Hamiltonian up to closed zero-form that is a constant $H_{\xi}^{0}$.

Note II.3. It is important to remember that this formula was obtained by treating $S^{\alpha}$ as boundary of $T^{\alpha}$. Therefore $\mathcal{B}_{(C)}$ is constructed as a boundary form for the time-like component of the boundary.

To recapitulate, as long as (2) and (8) make sense, they are all we need to construct explicitly a Hamiltonian for $\xi$ in a fully covariant, first order formulation. The Hamiltonian $H_{\xi}$ stems from the existence of a finite quasi-local tensor, thus that notion is underlying the derivation in an essential way but the tensor itself is not explicitly used. 
Note II.4. We have taken for granted that the only boundary the spacelike hypersurfaces $\Sigma$ have is a surface at large radial distances. In case this is not true an analogous condition (8) and corresponding additional terms in $H_{\xi}$ must be included, a procedure not really well-defined near actual singularities. This is a crucial detail. In what follows we would like to have the spatial infinity as the only boundary of $\Sigma$. For that to be so, we shall assume from now on that singular energy distributions implied by the metrics we write down are replaced by smooth ones such that no timelike singularities arise.

The value of the 'angular' integral over $S^{\infty}$ in (21) cannot be shifted by addition of local term on the boundary action terms. The phase space constant $H^{0}$ cannot be shifted. Its presence makes the generator potentially consistent with other constructions of the generators but it is a quantity without much meaning when we discuss quasi-local generators. Thus we shall give a name to the 'angular' integral. We will refer to it as the Brown-York generator:

$$
H=H_{B Y}+H^{0} .
$$

\section{Quasi-local generators and conservation}

We mentioned already in the introduction that a major problem in gravity is localizing energy. All our formal choices might appear natural from certain points of view, but they are essentially motivated by proposals addressing the localization problem. Equivalence principle or general covariance say that any energy density must vanish.

Going to the boundary was the brilliant move of Brown and York [5]. It fits nicely in 't Hooft's 'holography' idea: gravitational degrees of freedom are fundamentally boundary degrees of freedom 10]. There is a host of quasi-local definitions, see e.g. Ref. [35] and the works discussed there. Misner-Sharp mass [36] is another definition of mass of quasi-local type, applying in spherical symmetry, with nice properties 37]; for a Lovelock gravity it was defined recently in [38].

In our derivation we encountered the quantities

$$
H_{\xi}(\alpha)=\int_{S^{\alpha}}\left\{i_{\xi} \omega \cdot \frac{\partial \mathcal{L}}{\partial \Omega}+i_{\xi} \mathcal{B}_{(C)}\right\}+H_{\xi}^{0}
$$

What is important about them is that they converge to the canonical generator $H_{\xi}$ for $\alpha \rightarrow \infty$, where $r_{\alpha}$ is the radius of the hypersurface $T^{\alpha}$. One may regard $H_{\xi}(\alpha)$ as the value of the associated charge contained in the spatial sections bounded by $T^{\alpha}$.

Though this is not precise, as we will discuss in section VIII there is a reason for thinking something like that. $H_{\xi}(\alpha)$ is essentially the quasi-local mass of Brown and York, in first order formalism; their mass is defined as a canonical generator in the sense of Hamilton-Jacobi theory [5] [12]. In order to understand the claim, let $T^{\alpha}$ be a timelike hypersurface and $\zeta$ be a vector field tangential to it. $\zeta$ is not a Killing vector. By manipulations learned in the previous section it is straightforward to show that

$$
\begin{aligned}
& \int_{T^{\alpha}} d\left\{i_{\zeta} \omega \cdot \frac{\partial \mathcal{L}}{\partial \Omega}+i_{\zeta} \mathcal{B}_{(C)}\right\}= \\
& \int_{T^{\alpha}}\left\{£_{\zeta} \omega \cdot \frac{\partial \mathcal{L}}{\partial \Omega}+£_{\zeta} \mathcal{B}_{(C)}-i_{\zeta}\left(\mathcal{L}+d \mathcal{B}_{(C)}\right)+i_{\zeta} \Psi \cdot \mathcal{E}\right\}
\end{aligned}
$$

The last term vanishes on-shell, $\mathcal{E}=0$. The middle term vanishes as $\zeta$ is tangent on $T^{\alpha}$. Now, note first that the remaining term is nothing but the boundary term on $T^{\alpha}$ of the action functional for Lie derivative variations of the fields, see (4). Also we see from (10) that this reads $£_{\zeta} \Psi \cdot \Theta_{\text {quasi-local }}$. From precisely this term the quasi-local densities are read off from in the Hamilton-Jacobi analysis of Refs. [5] [12]. Now, the l.h.s. of the equation is nothing but the difference of $H_{\zeta}(\alpha)$ evaluated in the future and past $S^{\alpha}$. In other words, we obtain explicitly that the integrand involving the Brown-York quasi-local densities is a total derivative producing $H_{\zeta}(\alpha)$ on the boundary sphere. Thus, as mentioned in the introduction, $H_{\zeta}(\alpha)$ is essentially the canonical generator in the sense of Hamilton-Jacobi theory.

Conservation is easily obtained from the previous formula if we let $\zeta$ be a Killing vector: then the future and past values of $H_{\zeta}(\alpha)$ must agree. The time-derivative of (23) can also be calculated directly. Let a $\chi$ be a time-like vector field tangential on $T^{\alpha}$. By $\partial S^{\alpha}=0$ the previous formula tells us that the rate of change $\dot{H}_{\xi}(\alpha)$ of the quasilocal charge $H_{\xi}(\alpha)$ under the flow of $\chi$ reads:

$$
\begin{aligned}
\dot{H}_{\xi}(\alpha)=\int_{S^{\alpha}} & \left\{i_{\chi}\left\{£_{\xi} \omega \cdot \frac{\partial \mathcal{L}}{\partial \Omega}+£_{\xi} \mathcal{B}_{(C)}\right\}\right. \\
& \left.-i_{\chi} i_{\xi}\left(\mathcal{L}+d \mathcal{B}_{(C)}\right)+i_{\chi}\left(i_{\xi} \Psi \cdot \mathcal{E}\right)\right\}
\end{aligned}
$$

For any Killing vector $\xi$ we have that $£_{\xi} \Psi=0$ thus the first term should vanish. (If $\xi$ is an asymptotic Killing vector then conservation holds in the limit $\alpha \rightarrow \infty$.) If $\xi$ is a time-like Killing vector the remaining term vanishes for $\chi=\xi$ by the identity $i_{X} i_{X}=0$. (In general, the inner product operator satisfies: $i_{X} i_{Y}=-i_{Y} i_{X}$ for any vector fields $X$ and $Y$.) This a remnant of the 'commutativity' of Hamiltonian with itself. Mass and angular momentum as given by (23) are indeed conserved on-shell.

\section{Different boundary conditions}

A question which arises is: To what extent the Hamiltonian depends on the conditions $C$ ? To answer that one should look at the associated no-leaking condition, equation (8), which we may call it $F(C)$. The phase space is defined such that this condition holds. Let us denote by $C_{1}$ and $C_{2}$ two different boundary conditions. one works in two phase spaces where the conditions $F\left(C_{1}\right)$ and $F\left(C_{2}\right)$ hold. Two things may happen. One, the phase spaces have a measure zero intersection. Then we may obtain two different Hamiltonians. Second, the intersection of phase spaces 
is nice subset of each one. Then we may restrict our variations in there. Subtracting the 'no-leaking' conditions (8) we have that $i_{T^{\alpha}}^{*}\left(\boldsymbol{\delta} \mathcal{B}_{\left(C_{1}\right)}-\boldsymbol{\delta} \mathcal{B}_{\left(C_{2}\right)}\right)$ goes to zero for $\alpha \rightarrow \infty$. The previous derivation shows that the two Hamiltonians (21) associated with $C_{1}$ and $C_{2}$ may differ only by a phase space constant. For example, a set of counter-terms applying to asymptotically $A d S$ spacetimes were constructed and applied in Refs. [39 44 as boundary terms respecting the conformal symmetry of $A d S$ boundary. Though those counter-terms differ a lot from the simple boundary forms we are using the results are expected to agree and they indeed do as we shall see in examples. Additionally the same boundary forms apply straightforwardly with little formal modification to asymptotically de Sitter spacetimes, along the lines of Ref. 7]. From the point of view of Refs. [39 44] it is not clear why this is so; from the point of view of the Dirichlet counter-terms it is not that surprising.

\section{E. The Hamiltonian generator in type (II)}

When spacetime is asymptotically flat, there is no length scale to facilitate regularization. It is then characteristically difficult to construct a boundary form $\mathcal{B}_{(C)}^{\text {reg }}$ that would lead to finite charges. On the other hand, in this case the ADM construction of charges [3] it is a consistent method for calculating the energy and the angular momentum for asymptotically flat space. An extension of this is the Abbott-Deser mass [4] which calculates total energy for asymptotically de Sitter spacetimes.

From the point of view of first order formulation these are all subtraction methods. Energy and any charge is measured relatively to background by studying and formulating things in terms of the asymptotic deviations from the background.

In such cases the form $\mathcal{B}^{\text {reg }}$ should exist but one cannot find a local expression of it, or doesn't care to find one. It is not an accident that this applies to asymptotically flat spaces: the 'holographic renormalization' does not work straightforwardly in asymptotically flat spacetimes and the 'corrections' need to be non-local [45]. In specific case on though may find local but not polynomial counterterms which suffice to remove divergencies but they do not apply to all asymptotically flat spaces 24 27]. For the general case, non-locality suggests that the boundary form can be constructed by involving another solution of the equations of motion i.e. another point in phase space.

Let $\Psi$ and $\Psi_{\infty}$ be two solutions of the the equations of motion. The indication ' $\infty$ ' is simply a name to distinguish the second solution, its meaning will be explained below. Then subtracting conditions (77) applied on each solution we have:

$$
\left(\delta \omega \cdot \frac{\partial \mathcal{L}}{\partial \Omega}+\delta \mathcal{B}_{(C)}\right)-\left(\delta \omega \cdot \frac{\partial \mathcal{L}}{\partial \Omega}+\delta \mathcal{B}_{(C)}\right)_{\infty} \rightarrow \delta B
$$

pulled back into $T^{\alpha}, \alpha \rightarrow \infty$.

$B$ is a phase space function which is antisymmetric in fields $\Psi$ and $\Psi_{\infty}$. B must be annihilated by $\delta_{(C)}$, that is, $\delta B=\delta B\left(i^{*} \Psi, i^{*} \Psi_{\infty}\right)$. This is an important fact: Choose a field $\Psi_{\alpha}$ to induce the same field with $\Psi$ into $T^{\alpha}$ :

$$
i_{T^{\alpha}}^{*} \Psi=i_{T^{\alpha}}^{*} \Psi_{\alpha}
$$

such that there is a well defined limit $\Psi_{\alpha} \rightarrow \Psi_{\infty}$ as $\alpha \rightarrow \infty$. Then by antisymmetry $\delta B=0$ i.e.

$$
\boldsymbol{\delta} \omega \cdot \frac{\partial \mathcal{L}}{\partial \Omega} \rightarrow-\delta \mathcal{B}_{(C)}+\left(\boldsymbol{\delta} \omega \cdot \frac{\partial \mathcal{L}}{\partial \Omega}+\delta \mathcal{B}_{(C)}\right)_{\infty}
$$

pulled back into $T^{\alpha}, \alpha \rightarrow \infty$. Condition (27) is not necessary but allows us solubility; without it we are simply back to the general (26).

Let find now an explicit form for the $\infty$-part of the r.h.s. of (28) which we know is $\boldsymbol{\delta}$-exact. Let $\Psi_{\infty}$ be any solution that can be continuously related to $\Psi$ by the parameters. We have $\left(\boldsymbol{i}_{£_{\xi} \Psi} \boldsymbol{\sigma}\right)_{\infty}=-\boldsymbol{\delta} H_{\infty, \xi}$. Then relation (18) applied to $\Psi_{\infty}$ allows to write the r.h.s. of (28) as $\delta$-exact, at least under the integral sign in the process of the derivation of $H_{\xi}$. This is all one needs. Applying (28) in the derivation at the step of (18) one finds easily

$$
\begin{aligned}
H_{\xi}=\int_{S^{\infty}}\{ & i_{\xi} \omega \cdot \frac{\partial \mathcal{L}}{\partial \Omega}+i_{\xi} \mathcal{B}_{(C)} \\
& \left.-\left(i_{\xi} \omega \cdot \frac{\partial \mathcal{L}}{\partial \Omega}+i_{\xi} \mathcal{B}_{(C)}\right)_{\infty}\right\}+H_{\infty, \xi}
\end{aligned}
$$

The case $\Psi=\Psi_{\infty}$ tells us that the $\delta$-integration constant is zero. This is our Hamiltonian for the cases (II) e.g. when spacetimes are asymptotically flat. It is derived under the condition (7) in field space and condition (27) for the second field $\Psi_{\infty}$.

Unlike the constant $H_{\xi}^{0}$ in (21), $H_{\infty, \xi}$ is not a constant function in phase space. It is the value at of the Hamiltonian at $\Psi_{\infty}$. Now $\boldsymbol{\delta} H_{\infty, \xi}$ does not vanish unless $\Psi_{\infty}$ is equilibrium point of the phase space. This is true if $\Psi_{\infty}$ is an actual 'ground state' of the system. Of course in any case the $H_{\xi}$ satisfies (12) by construction.

Condition (27) is crucial for applications of (29). In general it cannot be satisfied if the fields are thought of as components w.r.t. the same coordinates. The induced fields will agree on $T^{\alpha}$ as geometric objects up to necessary diffeomorphisms. There is nothing unnatural in that when using manifestly invariant quantities. A condition of the form (27) was introduced in [46] as a means to guaranty finite-ness of a 'physical' action $S(\Psi)-S\left(\Psi_{\infty}\right)$, dragging also the conserved quantities to finite-ness. Here it was introduced as a convenient step integrating a Poincare invariant symplectic form in phase space.

We can now give a specific definition of the relative (onshell values of the) generators, first mentioned in definition II.1

Definition II.5. Every case can be regarded as type (II). So may bypass the arbitrariness of $H_{\infty}$ or use the arbitrariness of $H^{0}$ in type $(\mathbf{I})$ to define a relative generator $H_{\text {rel }}$ such that: $H_{\text {rel }}=0$ if a certain condition holds. The condition usually expresses the fact that we measure the charges such that their vacuum values are set to zero. 


\section{SPHERICAL AND 'TOPOLOGICAL' METRICS}

We start by studying some characteristic examples in three, four and five spacetime dimensions in Einstein gravity. Some interesting things arise when the angular manifolds are not spheres. Einstein gravity will be described by the Lagrangian

$$
\mathcal{L}=\frac{1}{2} c_{1} \epsilon(\Omega \underbrace{E \cdots E}_{D-2})=\frac{1}{2} c_{1} \epsilon\left(\Omega E^{D-2}\right)
$$

where $(D-2) ! c_{1}=(8 \pi G)^{-1}$, where $G$ we define as Newton's constant in the given dimensions, following the most standard conventions.

The standard form of the metrics we consider is

$$
d s^{2}=-g^{2} d t^{2}+\frac{d r^{2}}{g^{2}}+r^{2} d \Omega^{2}
$$

and $d \Omega^{2}$ is the metric of a compact i.e. closed, constant curvature co-dimension 2 manifold. Let a frame $\tilde{E}^{i}$ over this manifold i.e. $d \Omega^{2}=\delta_{i k} \tilde{E}^{i} \otimes \tilde{E}^{k}$ and $\tilde{\omega}^{i}{ }_{j}$ a connection. Its curvature will be $\tilde{\Omega}^{i j}=k \tilde{E}^{i} \tilde{E}^{j}, k= \pm 1,0$. Of course $k=1$ refers to the spherically symmetric case. But interesting facts are related to comparison of the that case to the flat $(k=0)$ manifold. The black holes associated with $k$ different than one have been termed as 'topological' (see below).

Let the frame

$$
E^{0}=g d t, \quad E^{1}=g^{-1} d r, \quad E^{i}=r \tilde{E}^{i} .
$$

Then of course $d s^{2}=\eta_{A B} E^{A} \otimes E^{B}$. As long as torsion is zero, and this always the case for Einstein gravity in vacuum, we have that the non-zero components of the connection are

$$
\omega_{1}^{0}=g g^{\prime} d t, \quad \omega_{1}^{i}=g \tilde{E}^{i}, \quad \omega_{j}^{i}=\tilde{\omega}_{j}^{i} .
$$

The first one we would like to analyze is the most standard of all: four dimensional Schwarzschild metric with zero cosmological constant. Then

$$
g^{2}=1-\frac{2 G m}{r}
$$

We would like to analyze how $m$ arises as the total energy of the system.

Energy, or mass, will be calculated as the on-shell value of the Hamiltonian for the Killing vector $\xi=\partial / \partial t$. The problem, due to asymptotic flatness, is case (II) as one may check for oneself. We use (29).

For Einstein gravity and Dirichlet boundary conditions the boundary form is $\mathcal{B}_{(C)}=-\frac{1}{2} c_{1} \epsilon(\theta E E)$, as discussed in the appendix. The form $\theta=\omega-\omega_{\|}$is constructed from the induced connection $\omega_{\|}=i^{*} \omega^{a b}$ of a constant $r=$ $r_{\alpha}$ hypersurface $T^{\alpha}$, where $a, b=0, i$. [The induced and intrinsic fields equalities, for the connection as well as $E_{\|}=$ $i^{*} E$, are consistent for zero intrinsic torsion.]
Thus we have $\theta^{01}=g g^{\prime} d t$ and $\theta^{i 1}=g \tilde{E}^{i}$. The first part of the integral in (29) reads

$$
\int_{S^{2}} c_{1} \cdot\left(\frac{1}{2} \epsilon\left(i_{\xi} \omega E E\right)-\frac{1}{2} i_{\xi} \epsilon(\theta E E)\right)=-\frac{1}{G} g^{2} r,
$$

evaluated at $r=r_{\alpha} \rightarrow \infty$ as $\alpha \rightarrow \infty$. This term of (29) alone diverges. Before proceeding we digress for a couple of comments.

One should note that there are no derivatives in the result. The divergence is due to this fact. This means that the 'no-leaking' condition is not respected thus (35) is not a final result. Nonetheless, we may note that in fact all our final formulas will ot contain derivatives of the metric function.

Ref. [47] seems to have a general setting similar to ours. Various ingredients e.g. first order formalism, boundary forms, covariant phase space are there. The results though disagree, the Hamiltonian is convergent by construction. The difference lies in the definition of the phase space. [47] imposes conditions on the fields associated to asymptotic flatness. Without them certain ambiguities of the asymptotic Poincare group i.e of the energy-momentum definitions arise in four dimensions. Doing so, one again implicitly works with Minkowski as reference spacetime from the very beginning, trying to make sense of the formulas under that condition. Moreover, the asymptotic conditions on $\omega$ are imposed using torsion-free-ness. This brings the analysis closer to the second order formalism. In all, the results of Ref. [47] are expected to be very different from ours. The ambiguities themselves are a matter of geometry first of all, and any set of requirements on the fields which remove them could be applied when a definition of energy has been adopted. We will not go into these issues here.

We introduce fields $\Psi_{\alpha}$ such that the condition (27) holds: Let

$$
d s_{\alpha}^{2}=-g_{\alpha}^{2} d t_{\alpha}^{2}+\frac{d r_{\alpha}^{\prime 2}}{g_{\alpha}^{2}}+r_{\alpha}^{\prime 2} d \Omega^{2},
$$

where $g_{\alpha}=g_{\alpha}\left(r_{\alpha}^{\prime}\right)$ is another solution, say for the parameter $m_{\alpha}$. The change of coordinates is given by $r=r_{\alpha}$, $r_{\alpha}^{\prime}=r_{\alpha}$ and $g\left(r_{\alpha}\right) t=g_{\alpha}\left(r_{\alpha}\right) t_{\alpha}$ for some fixed radius $r_{\alpha}$. Thus (27) hold.

What in particular (27) means is that

$$
\xi=\frac{\partial}{\partial t}=\frac{g}{g_{\alpha}} \frac{\partial}{\partial t_{\alpha}} .
$$

Thus the integral in the Hamiltonian (29) equals

$$
-\frac{1}{G}\left(g^{2} r-g g_{\alpha} r\right),
$$

everything evaluated at $r_{\alpha}$ which goes to infinity. This is convergent and we have $H_{\xi}=m-m_{\infty}+H_{\infty, \xi}$. In particular we may use Minkowski spacetime fields $m_{\infty}=0$ as the $\Psi_{\infty}$ phase space point. As mentioned in the previous section, for the asymptotically non trivial Killing $\xi$ the corresponding canonical generators are tensorial quantities 
of the asymptotic symmetries. In particular as long as these symmetries indeed are Lorentz transformations then $H_{\infty, \xi}=0$ that is $H_{\xi}=m$ indeed.

Analyzing how the result arises one observes something interesting. The finite part of (35) is $2 \mathrm{~m}$. The divergent part is due to the angular manifold (2-sphere) constant curvature: $\tilde{\Omega}^{i j}=1 \cdot \tilde{E}^{i} \tilde{E}^{j}$. Consider now the more general case given by (36) i.e. $k= \pm 1,0$. Then the field equations are equivalent to $\left(r g^{2}\right)^{\prime}=k$, that is

$$
g^{2}=k-\frac{r_{0}}{r},
$$

where $r_{0}$ is an integration constant. These solutions are the zero cosmological constant analogues of the $A d S$ topological black holes [48] [49] 50].

When $k=0$ the two-manifold is flat. It can be a torus or a Klein bottle. Its volume $v o l_{0}$ is arbitrary. In that case (35) converges. That is the problem is a type $(\mathbf{H})$ case. The Brown-York mass reads:

$$
k=0: \quad m_{B Y}=\frac{v o l_{0}}{4 \pi} \frac{r_{0}}{G} .
$$

Let's re-calculate the mass as a relative mass applying the type II) Hamiltonian (29) which here reads (38). Let $g_{\alpha}$ be such that $r_{0}(\alpha) \rightarrow 0$ for $\alpha \rightarrow \infty$. Clearly the result is again (40). Thus we indeed deal with a relative mass which can also be regarded as a Brown-York mass, in accordance with the definition $\amalg .1$ of the type $(\mathbf{H})$ case.

This phenomenon persists even in the quite more complicated case of Einstein-Gauss-Bonnet gravity as we will see. This is due to the fact that $g_{\alpha} \rightarrow 0$ for the specific solution. There is a finite discontinuity going from the $A d S$ metrics of length scale $l$ to their $l=\infty$ analogues when they are $(\mathbf{H})$ metrics, as we shall see.

Let us turn to study anti de Sitter vacuum metrics in three, four and five dimensions, in the presence also of mass. An anti de Sitter vacuum means that the equations of motion read $\epsilon\left(\left\{\Omega+l^{-2} E E\right\} E^{D-3} \delta E\right)=0$, that is the bulk Lagrangian is

$$
\mathcal{L}=\frac{1}{2} c_{1} \epsilon\left(\Omega E^{D-2}\right)+\frac{1}{2} c_{1} \frac{D-2}{l^{2} D} \epsilon\left(E^{D}\right) .
$$

Again $(D-2) ! c_{1}=(8 \pi G)^{-1}$.

The three dimensional pure anti de Sitter spacetime is of the form (36), explicitly

$$
d s^{2}=-\left(1+\frac{r^{2}}{l^{2}}\right) d t^{2}+\left(1+\frac{r^{2}}{l^{2}}\right)^{-1} d r^{2}+r^{2} d \phi^{2} .
$$

The vielbein is $E^{0}=g d t, E^{1}=g^{-1} d r, E^{2}=r d \phi$, and the non-zero components of the connection read $\omega^{0}{ }_{1}=g g^{\prime} d t$, $\omega^{2}{ }_{1}=g d \phi$.

We first calculate

$$
\int_{S^{1}} c_{1} \cdot\left(\frac{1}{2} \epsilon\left(i_{\xi} \omega E\right)-\frac{1}{2} i_{\xi} \epsilon(\theta E)\right)=-2 \pi c_{1} g^{2},
$$

for $\xi=\partial / \partial t$. This expression diverges

$$
-2 \pi c_{1} \frac{r^{2}}{l^{2}}-2 \pi c_{1} .
$$

The problem is type (I) case. The previous result was calculated with the minimal Dirichlet boundary form, discussed in the appendix. Let a boundary form as

$$
\mathcal{B}_{(C)}=-\frac{1}{2} c_{1} \epsilon(\theta E)+\frac{1}{2} c_{1} b_{0} \epsilon\left(E_{\|} E_{\|}\right)_{1} .
$$

Note III.1. $\epsilon(\cdots)_{1}$ shall mean from now on contraction with the volume form $\epsilon_{a \ldots b 1}$ on the intrinsic Lorentz bundle; it is identified with the induced 'bulk' volume form, where $E^{1}$ in practice is always the radial co-vector. A theoretical problem is that $\epsilon_{a \ldots b 1}$ is not invariant under variations, potentially contributing terms inexistent in our derivations. This is not so. Let $\zeta$ a unit vector, $\eta_{A B} \zeta^{A} \zeta^{B}=1$, normal on the boundary. That is $\epsilon_{a \ldots b 1}=\epsilon_{a \ldots b A} \zeta^{A} . \quad \delta \zeta^{A}$ is not zero but the unit length of $\zeta$ implies that $\delta \zeta^{A}$ is normal to $\zeta^{A}$ i.e. tangential. As a result it drops out the variations of the intrinsic boundary forms as it contributes one tangential index too many.

The result (43) is 'corrected' by $-2 \pi c_{1} b_{0} g r$ which asymptotically reads

$$
-2 \pi c_{1} b_{0} \frac{r^{2}}{l}-\pi c_{1} b_{0} l
$$

Canceling the divergence fixes $b_{0}=-l^{-1}$. So finally we have

$$
m_{B Y}=-c_{1} \pi=-\frac{1}{8 G} .
$$

Expression (47) is a well known result.

For the Poincare patch of the $A d S_{3}$

$$
-\frac{r^{2}}{l^{2}} d t^{2}+\frac{l^{2}}{r^{2}} d r^{2}+r^{2} d \phi^{2}
$$

one similarly finds the correct answer $m_{B Y}=0$. Things work nicely. The three dimensional case will be discussed and commented on in detail in the next section.

A four dimensional anti de Sitter Schwarzschild solution is given the $k=1$ case of (36) with

$$
g^{2}=\frac{r^{2}}{l^{2}}+k-\frac{r_{0}}{r} .
$$

where $l$ is the $A d S$ radius and $r_{0}$ an integration constant. We will consider the general $k$. This is a case $(\mathbf{I})$ and we apply (21) for a (non-minimal) boundary form:

$$
\mathcal{B}_{(C)}=\frac{1}{2} c_{1}\left\{-\epsilon(\theta E E)+b_{0} \epsilon\left(E_{\|}^{3}\right)_{1}+b_{1} \epsilon\left(\Omega_{\|} E_{\|}\right)_{1}\right\} .
$$

The constants are to be determined by finiteness. The Hamiltonian is given by the result (35) plus $b$-'corrections':

$$
-16 \pi c_{1} g^{2} r+12 \pi c_{1} b_{0} g r^{2}+4 \pi c_{1} b_{1} k g,
$$


times a factor $\operatorname{vol}_{k} /(4 \pi)$ where $v l_{k}$ is the volume of the 2manifold. The expression above is evaluated at a constant $r=r_{\alpha}$ which goes to infinity. The divergencies are canceled for $b_{0}=4 /(3 l)$ and $b_{1}=2 l$. $b_{1}$ is useless and not defined for $k=0$. The Brown-York Hamiltonian turns out to be

$$
m_{B Y}=\frac{\operatorname{vol}_{k}}{4 \pi} \frac{r_{0}}{2 G} .
$$

This holds for any $k$ and regularization is required in all cases.

For the spherical case $k=1$ this is the well known Schwarzschild mass we found in the asymptotically flat case, formally $l \rightarrow \infty$. No discontinuity arises between the case for finite $l$ and $l \rightarrow \infty$. Presumably, one may conclude, no energy can be associated with the vacuum itself arises in four dimensions.

Now for the flat angular 2-manifold i.e. $k=0$ things are different. The result (40) in the asymptotically flat case $l=\infty$ studied above is twice the result we find now for finite $l$.

Remark III.2. In a case $(\mathbf{H})$, where no regularization is needed for finintness, the mass is twice the mass one finds when the metric is looked at in the presence of an intrinsic length, as it happens in the presence of the cosmological constant. [It will turn out that the remark holds as long as the Einstein term is present in the Lagrangian.]

Let's see how things work out in the one dimension higher than the usual macroscopic dimension of spacetime. The five dimensional anti de Sitter Schwarzschild solution is a case where, as in three dimensions, is turns out that the energy of vacuum is not zero. The difference w.r.t. three dimensions is that vacuum energy is not convergent for $l \rightarrow \infty$.

Let the metric be given by the five-dimensional analogue of (36) with

$$
g^{2}=\frac{r^{2}}{l^{2}}+k-\frac{r_{0}^{2}}{r^{2}}
$$

i.e. the angular three manifold has constant curvature $k$. The Hamiltonian will be given by

$$
\int_{S^{3}}\left(\frac{1}{2} c_{1} \epsilon\left(i_{\xi} \omega E^{3}\right)+\mathcal{B}_{(C)}\right),
$$

for a boundary form

$$
\mathcal{B}_{(C)}=\frac{1}{2} c_{1}\left\{-\epsilon\left(\theta E^{3}\right)+b_{0} \epsilon\left(E_{\|}^{4}\right)_{1}+b_{1} \epsilon\left(\Omega_{\|} E_{\|}^{2}\right)_{1}\right\} .
$$

The result is

$$
-36 \pi^{2} c_{1} g^{2} r^{2}+24 \pi^{2} c_{1} b_{0} g r^{3}+12 \pi^{2} c_{1} b_{1} k g r,
$$

times $\operatorname{vol}_{k} /\left(2 \pi^{2}\right)$. Divergencies cancel for

$$
b_{0}=\frac{3}{2 l}, \quad b_{1}=\frac{3 l}{2},
$$

where again $b_{1}$ is useless and not defined for $k=0$, and the finite Brown-York mass is

$$
18 \pi^{2} c_{1}\left(\frac{k^{2} l^{2}}{4}+r_{0}^{2}\right) \frac{\operatorname{vol}_{k}}{2 \pi^{2}} .
$$

For the usual definition $3 ! c_{1}=(8 \pi G)^{-1}$ this agrees with the known results in the spherically symmetric case [6] [51].

The couplings $b_{0}$ and $b_{1}$ satisfy the relation

$$
-l^{2} \frac{d b_{0}}{d l}=\frac{d b_{1}}{d l} .
$$

We will see that when Einstein gravity is supplemented by the Gauss-Bonnet term, the dependence of $b_{0}$ and $b_{1}$ on $l$ changes but relation (59) holds as it is.

Clearly this result is hopeless in the limit $l \rightarrow \infty$. For comparison purposes, consider the pure Schwarzschild metric $g^{2}=1-r_{0}^{2} r^{-2}$. This is a case (II). We apply (29) and following steps explained at the beginning of this section we find that the relative mass is given by the limit of

$$
-36 \pi^{2} c_{1}\left(g-g_{\alpha}\right) g r^{2}+m_{r_{0}=0}
$$

where we imply that the fields $\Psi_{\infty}$ are the Minkowski solution $r_{0}=0$. We have

$$
m=18 \pi^{2} c_{1} r_{0}^{2}+m_{r_{0}=0} .
$$

By Lorentz invariance the pure Minkowski spacetime energy should vanish, $m_{r_{0}=0}=0$.

In all, formula (58) can be thought of as describing a Schwarzschild mass given by the previous result, and a vacuum energy given by

$$
m_{B Y}=-\frac{3 \pi}{32 G K},
$$

where we wrote $c_{1}$ in terms of the five dimensional Newton's constant and $K=-l^{-2}$ is the constant curvature of spacetime.

We will see the result (62) appearing again when we consider Lovelock gravity in dimension five. It will only be corrected by a term depending only on the gravitational couplings, analogous to the three dimensional vacuum energy.

The vacuum energy (62) is divergent for infinite $l$. On the other hand the case $l=\infty$, that is asymptotically flat, is not one such that the relative mass is a Brown-York mass i.e. is not type $(\mathbf{H})$ and the remark III.2 need not apply.

\section{POINT PARTICLE IN THREE DIMENSIONS}

The asymptotically flat (locally) three dimensional spherical metric has been bypassed up to this point. It belong to the hybrid case, type $(\mathbf{H})$, and we shall apply remark ПI.2 We shall discuss it at some length.

Einstein gravity in three dimensions is an always intriguing problem. It is because it feels very elementary in some sense and to an unimaginable extent soluble. In three 
dimensions there is no curvature outside matter, i.e. no gravity in vacuum hence no propagation of gravity through gravitational waves. In other words the effect of gravitational sources is not local, they rather affect spacetime in a global manner.

One implication of this is that singularities are integrable. On the other hand all of the discussion of Hamiltonians that so emphasizes spatial infinity is in the spirit that the gravitational effects are diminishing there. This is not the case in three dimensions, the deficit angle does not seize to exist for large distances. Nevertheless things seem to work and it is interesting to apply the formulas anyway.

Interest in Einstein gravity in three spacetime dimensions was essentially initiated by 52 .

Einstein gravity in three dimensions without cosmological constant can be described by the Lagrangian

$$
\mathcal{L}=\frac{1}{2} c_{1} \epsilon(\Omega E)
$$

$c_{1}=(8 \pi G)^{-1}$. The field equations say that torsion $T^{A}=0$ and curvature $\Omega^{A B}=0$.

The metric

$$
d s^{2}=-(d t+a d \phi)^{2}+\frac{d r^{2}}{\beta^{2}}+r^{2} d \phi^{2},
$$

is a spherically symmetric solution. $0<r<\infty$ and $0<$ $\phi<2 \pi . \beta$ and $a$ are constants.

From the vielbein

$$
E^{0}=d t+a d \phi, \quad E^{1}=\frac{d r}{|\beta|}, \quad E^{2}=r d \phi
$$

$T^{A}=0$ gives the connection

$$
\omega_{1}^{2}=|\beta| d \phi
$$

Thus $\Omega=0$ and both field equations are satisfied.

Space can be given in conformally flat coordinates. Let $r=\rho^{1-\gamma}$. Then

$$
d s^{2}=-(d t+a d \phi)^{2}+\rho^{-2 \gamma}\left(d \rho^{2}+\rho^{2} d \phi^{2}\right),
$$

where $\beta^{2}=(1-\gamma)^{2}$. The interesting thing here is the origin $r=0$ is mapped to the origin $\rho=0$ if $\gamma<1$ and to $\rho=\infty$ if $\gamma>1$. Also the metric is invariant under the transformation $\rho=1 / \rho^{\prime}$ and $\gamma=2-\gamma^{\prime}$. The other coordinate system is not transformed: $r^{\prime}=r$ and $\left|\beta^{\prime}\right|=|\beta|$.

So in some sense $\rho=0$ is dual to $\rho=\infty$. There is a difference: if $\gamma>1, \rho=0$ lies at infinite proper distance, $\int_{0}^{\rho_{0}} d \rho \rho^{-\gamma}=\infty$, from any point $\rho_{0}$. Instead $\rho=\infty$ lies at finite proper proper distance from an arbitrary point, $\int_{\rho_{0}}^{\infty} d \rho \rho^{-\gamma}<\infty$

Let's calculate first the mass. It is the Hamiltonian for the Killing vector is $\xi=\partial / \partial t$. Its amusing to do that in both kinds of coordinates.

As discussed one can always treat a case as type (II) and calculate a charge as a relative charge applying (29). We will do that for the moment. We chose $\Psi_{\infty}$ to be the metric with $\beta^{2} \rightarrow 1$. The crucial condition (27) is satisfied trivially here. $T^{\alpha}$ is the hypersurface at some fixed radius $r_{\alpha}$ in both radial coordinates we write $\Psi$ and $\Psi_{\alpha}$ (the need not be the same). Using (65) and (66) the first line of (29) equals

$$
\int_{S^{1}} c_{1} \cdot\left(\frac{1}{2} \epsilon\left(i_{\xi} \omega E\right)-\frac{1}{2} i_{\xi} \epsilon(\theta E)\right)=-\frac{1}{4 G}|\beta|,
$$

thus

$$
m=\frac{1}{4 G}(1-|\beta|)+m_{\beta=1} .
$$

The sphere at infinity is a unit circle $S^{1} . \quad \theta=\omega-\omega_{\|}$ is calculated for the constant radius hypersurface $r=r_{\alpha}$, where $r_{\alpha} \rightarrow \infty$ for $\alpha \rightarrow \infty$. This is the surface $T^{\alpha}$. Its $\omega_{\|}=0$. Note that $\theta$ is the second fundamental form only because by our derivation we have taken $T^{\alpha}$ to be such that $\xi$ is tangent to it.

Consider now the conformally flat space coordinates case. The spatial vielbein now is

$$
E^{1}=\eta \rho^{-\gamma} d \rho, \quad E^{2}=\rho^{1-\gamma} d \phi
$$

The connection is $\omega_{1}^{2}=\eta(1-\gamma) d \phi$.

$\eta= \pm$. A hypersurface at constant $\rho$ viewed as an outer boundary will have a normal vector in the direction of $E^{1}$. When $\eta=+$ it means that we take $E^{1}$ in the direction of growing $\rho$ i.e. towards infinity. When $\eta=-, E^{1}$ is in the direction of decreasing $\rho$ i.e. towards the origin of that coordinate.

The formula for the Hamiltonian is obtained treating $T^{\alpha}$ as an outer boundary. When $\eta=+$ we integrate around the origin of $\rho$ and when $\eta=-$ we integrate around the infinity of $\rho$.

When $\gamma<1$ the infinity of $\rho$ is at infinite proper distance from any point. We take $\Psi_{\infty}$ to be the metric for $\gamma \rightarrow 0$. Formula (29) gives

$$
m=\frac{1}{4 G} \gamma+m_{\gamma=0}
$$

When $\gamma>1$ the infinity of $\rho$ lies at finite distance from an arbitrary point $\rho_{0}$ while the origin is at infinite proper distance from it: Infinity is at $\rho=0$.

We may compactify the space, add a point at infinity, and have then two particles, one at an origin and one at infinity. Without compactification, the 'place' where a particle can be is at $\rho=\infty$ in these coordinates.

Integrating around $\rho=\infty$ means taking $\eta=-1 . \gamma>1$ so we cannot take $\Psi_{\infty}$ to the metric for $\gamma \rightarrow 0$. We will take the 'dual' choice, $\gamma \rightarrow 2$. Applying formula (29) we find now

$$
m=\frac{1}{4 G}(2-\gamma)+m_{\gamma=2}
$$

The two formulas we obtained are the two cases one obtains from (69) from the two solutions the relations of the parameters $\beta^{2}=(1-\gamma)^{2}$, and for the respective 'locations' 
one would expect form the radial transformation. This is of course due to general covariance.

For $\gamma=1$ both $\rho=0$ and $\rho=\infty$ lie at infinite proper distance from any $\rho_{0}$. It is the case of the space with the topology of the cylinder. The candidate 'places' for a particle are the infinities of a real line. These cannot be considered as locations of particles.

In [53] it was argued that in the case $\gamma \geq 1$ one cannot apply uniformly the asymptotic conditions one applies also for $\gamma<1$, because a (finite) Hamiltonian does not exist. One may think of that as a mathematical explanation in the canonical framework of the arguments given above.

Presumably, one may note that this discussion is hardly needed if we use the 'Schwarzschild' coordinates (64). The cylindrical case corresponds to $\beta=0$ which is already unacceptable in that form of the metric.

Let us now calculate the Hamiltonian generators using (21), that is as type (I). Let's use the minimal boundary form $\mathcal{B}_{(C)}=-\frac{1}{2} c_{1} \epsilon(\theta E)$. The result is essentially given by (68). We have

$$
m_{B Y}=-\frac{1}{4 G}|\beta|,
$$

The result is perfectly finite, without regularization, that is we deal with a type $(\mathbf{H})$ case.

Spacetime (64) is 'spinning'. Its angular momentum equals minus the Hamiltonian $H_{\xi}$ for the Killing vector $\partial / \partial \phi$. We have:

$$
J_{B Y}=-\int_{S^{1}} c_{1} \cdot\left(\frac{1}{2} \epsilon\left(i_{\xi} \omega E\right)-\frac{1}{2} i_{\xi} \epsilon(\theta E)\right)=\frac{1}{4 G}|\beta| a .
$$

The term involving $\theta$ originating from the boundary does not actually contribute, as the Killing vector is tangent onto the sphere. In terms of the quasi-local mass (73) it is simply expressed as

$$
J_{B Y}=-m_{B Y} a .
$$

There is a number of things that can be said here. Recall the definition 1 I.5 for the relative generators. Let's explicitly define a relative mass $m_{\text {rel }}$ by the condition: $m_{r e l}=0$ for $|\beta|=1$ i.e. for Minkowski spacetime. This fixes the free constant $H_{\xi}^{0}$. We have:

$$
m_{r e l}=\frac{1}{4 G}(1-|\beta|) .
$$

This the value calculated in [52] and in any calculation that integrates the matter energy tensor. Similarly one may define $J_{\text {rel }}$ by the condition: $J_{\text {rel }}=0$ if $a=0$. We have

$$
J_{r e l}=\left(\frac{1}{4 G}-m_{r e l}\right) a .
$$

Surprisingly relations (75) and (77) are apparently unknown in the literature. In Ref. [52] only the case $m_{r e l}=0$ is calculated, obtaining $a=-4 G J$. The general multiparticle massive and spinning metric was obtained in [54], but the integration constants were not carefully related to canonical generators.

Consider now the quasi-local mass $m_{B Y}$ for flat spacetime. For $|\beta|=1$ and $a=0$ we have:

$$
m_{B Y}=-\frac{1}{4 G}
$$

In accordance with remark III.2 this is indeed twice the vacuum energy (47) for $A d S$ spacetime.

Reference [55], reasoning essentially along the lines of formula (73), suggests that the vacuum energy of Minkowski spacetime in three dimensions is non-zero and equal to (78). On the other hand Ref. [53] using Regge-Teitelboim methods finds the result on the r.h.s. of (76), in agreement with 52], that is the Minkowski space mass is found to be simply zero. The results are not inconsistent: they differ by a phase space function which an arbitrariness of the definition anyway, relation (22). It does raise though issues but it is rather a matter of consistent application of definitions. If both kinds of definitions apply they should give equivalent physical results; if one of them is more relevant, for example in the general setting of $A d S / C F T$ ideas the Brown-York definition is the relevant one, the results should be taken seriously whatever they are. For example Minkowski spacetime should apparently be treated according to (78).

\section{FIVE DIMENSIONAL CHERN-SIMONS POINT PARTICLE}

We wish now to turn to the nearest analogue of the point particle in three dimensional gravity. If analogously to three dimensions we consider a Chern-Simons theory of the five dimensional Lorentz group we deal with a gravity that is described by a pure Gauss-Bonnet term. This is not exactly true unless we are in pure vacuum, but the analogies between three and five dimensions we will find, should be traced to the Chern-Simons equivalence of these gravities at the level of the Lagrangian.

Presumably, working in a first order formalism, we are able to construct Hamiltonians even in the presence of torsion. The following are an example of this fact.

Consider the Lagrangian

$$
\mathcal{L}=\frac{1}{2} c_{2} \epsilon(\Omega \Omega E)
$$

where $c_{2}$ is a coupling constant with dimensions of mass. In [56] it was observed that 'gravity' described by this Lagrangian admits a vacuum solution which is the obvious generalization of (64) for $a=0$, i.e. non spinning, and its (relative) mass was calculated.

We consider the obvious generalization of the full spinning metric (64):

$$
-(d t+a)^{2}+\frac{d r^{2}}{\beta^{2}}+r^{2} d \Omega^{2}
$$


where $a=a^{i} \tilde{E}^{i}$ for some Kerr constants $a^{i}$, and $d \Omega^{2}=$ $\delta_{i j} \tilde{E}^{i} \tilde{E}^{j}$ is the metric of the unit 3 -sphere, expressed conveniently in terms of a frame $\tilde{E}^{i}$. The frame satisfies the relation $d \tilde{E}^{i}=\epsilon_{i j k} \tilde{E}^{j} \tilde{E}^{k}$, where we may identify $\epsilon_{i j k} \equiv \epsilon_{01 i j k}$.

Now if by habit we insist that torsion is zero, then (80) does not solve the vacuum fields equations.

In Einstein gravity, when a form like $d t+a$ is introduced in the metric one assumes $d a=0$, precisely to avoid introducing torsion. We will impose:

$$
d a=T^{0} .
$$

Under this condition the zero torsion connection of the metric with $a^{i}=0$, that is $\omega^{i 1}=\beta \tilde{E}^{i}$ and $\omega^{i j}=\epsilon_{i j k} \tilde{E}^{k}$, is also a connection for (80), but with non-trivial torsion

$$
T^{0}=a^{i} \epsilon_{i j k} \tilde{E}^{j} \tilde{E}^{k}
$$

One should note two things. First, unlike the three dimensional Einstein case the metric (80) is not flat. The curvature reads: $\Omega^{i j}=\left(1-\beta^{2}\right) \tilde{E}^{i} \tilde{E}^{j}=\left(1-\beta^{2}\right) r^{-2} E^{i} E^{j}$. In fact there is a curvature singularity at $r=0$. There is also a torsion singularity there, $T_{i j}^{0}=a^{i} \epsilon_{i j k} r^{-2}$. The situation is somewhat analogous: $\delta$-function singularities in curvature and torsion in three dimensions [57] are replaced by $r^{-2}$ singularities in five.

Second, the torsion $T^{0}$ needs no source. The vacuum field equations are satisfied everywhere outside the singularity $r=0$. Also, just like in the three dimensional Einstein gravity, the singularity is integrable [56]. Put differently it is a case of type $(\mathbf{H})$.

We may verify that indeed no regularization is needed. The mass is calculated as the Hamiltonian (21) for the Killing vector $\xi=\partial / \partial t$ from the formula

$$
\int_{S^{3}} c_{2} \cdot\left(\epsilon\left(i_{\xi} \omega \Omega E\right)-i_{\xi} \epsilon\left(\theta\left\{\Omega_{\|}+\frac{1}{3} \theta^{2}\right\} E\right)\right) .
$$

The $\mathcal{B}_{(C)}$ form used is the well known boundary term for Dirichlet conditions constructed in Ref. [58] (see also the appendix). One finds easily that the quasi-local mass is given by

$$
m_{B Y}=-8 \pi^{2} c_{2}|\beta|\left(3-\beta^{2}\right) .
$$

This presumably gives a Brown-York vacuum for Minkowski spacetime in in this theory equal to

$$
m_{B Y}=-16 \pi^{2} c_{2} .
$$

It is the five dimensional analogue of the $-2 \pi c_{1}=-(4 G)^{-1}$ result of Ref. [55] for the three dimensions we encountered in the previous section.

Defining a relative mass similarly to the three dimensions by $m_{\text {rel }}=0$ for $\beta=1$ we obtain here

$$
m_{\text {rel }}=8 \pi^{2} c_{2}\left\{2-|\beta|\left(3-\beta^{2}\right)\right\} .
$$

This is the result found in [56] by integrating the matter energy tensor. It was shown that it can be calculated very elegantly using the Gauss-Bonnet theorem; the constant term, which is minus the alleged Minkowski vacuum energy, is related to the Euler number of a spatial 4-ball.

It perhaps interesting to note that, for $c_{2}>0$, the mass $m$ in this example is bounded from below, with a minimum at $\beta^{2}=1$. This is to be contrasted with the definite bounded-ness from above of (76) in three dimensions, which was especially discussed in Ref. [53]. In fact, the same happens in Chern-Simons theories in all dimensions which are multiple of four modulo one and three, respectively.

Now let $\xi^{i}$ be Killing vectors dual to $\tilde{E}^{i}$. The quasi-local angular momentum is calculated as minus the Hamiltonians, given by (83), for the vectors $\xi^{i}$. We have:

$$
J_{B Y}^{i}=8 \pi^{2} c_{2}|\beta|\left(1-\beta^{2}\right) a^{i} .
$$

It amusing to note the relation

$$
-3 J^{i}=\beta \frac{\partial}{\partial \beta} m a^{i},
$$

which also holds in three dimensions but with a factor -1 instead of -3 . Thus angular momentum is the scaling of the mass for the Chern-Simons particles. Relation (88) properly generalizes (75).

\section{BOULWARE-DESER-CAI METRICS IN $5 d$}

We now turn to the full Lovelock gravity in five dimensions. We study the spherically symmetric metrics of Boulware and Deser [59], discovered at the same time by J.T. Wheeler [60], and the generalization by Cai 61]. These are solutions to the so-called Einstein-Gauss-Bonnet gravity in five dimensions described by the Lagrangian

$$
\mathcal{L}=\frac{1}{2} c_{2} \epsilon(\Omega \Omega E)+\frac{1}{2} c_{1} \epsilon\left(\Omega E^{4}\right)-\frac{\lambda}{5 !} \epsilon\left(E^{5}\right) .
$$

The last term is formally equivalent to a 'matter' energy tensor $T_{B}^{A}=-\lambda \delta_{B}^{A}$. $\lambda$ is not an integration constant thus it is not a phase space parameter; it operates as one more gravitational coupling. For the Boulware-Deser-Cai solution is a vacuum solution for this Lagrangian everywhere outside the origin $r=0$ (which is not included in spacetime anyway). Torsion is assumed zero which solves the connection field equations.

Abbott-Deser type of calculations were done in 62 63 64 for quadratic curvature gravities. The resulting formulas compute the mass relative to the asymptotic vacuum. They are rather involved and hard to generalize. (The mass parameter in Boulware-Deser-Cai metrics given usually in the literature is calculated by these formulas.)

A basic feature of Lovelock gravity is that its solutions are multi-valued and its branches have different asymptotics. Relative mass depends on the reference background i.e. the asymptotic vacuum. Thus one cannot associate a value of energy with an entire solution. 
Brown-York computations are in no better shape in that respect; they too depend on asymptotics: the counterterms depend on the branch. On the other hand the BrownYork definition is more self-consistent: the branch of the solution we work at is already chosen at the level of the Lagrangian. Then a value of energy is obtained for the solution.

Stability analysis based on energy considerations 62 64 is treacherous in Lovelock gravity. The theory regards both branches as available states. The Brown-York definition emphasizes strongly that we work one branch at a time, there is no overview of the solution. The issue of stability of vacua was touched upon in Ref. [65]. We shall shown in a separate work that instabilities are far more generic in this gravity that what is envisaged in [65]. Here we shall point out that Brown-York masses make an unexpected appearance in semiclassical calculations. Quasi-local calculations in Lovelock gravity have been presented in various works, see e.g. 39 44, 66 72.

The metrics can be given the general form considered in section 【II.

$$
d s^{2}=-g^{2} d t^{2}+\frac{d r^{2}}{g^{2}}+r^{2} d \Omega^{2}
$$

$d \Omega^{2}$ is the metric of a compact, constant curvature $k$ threemanifold: denoting a frame on it by $\tilde{E}^{i}$ i.e. $d \Omega^{2}=\delta_{i k} \tilde{E}^{i} \otimes$ $\tilde{E}^{k}$ its curvature will be $\tilde{\Omega}^{i j}=k \tilde{E}^{i} \tilde{E}^{j}$, where $k= \pm 1,0$. The metric function $g^{2}$ is given by

$$
g^{2}-k=\frac{3 c_{1}}{2 c_{2}} r^{2}\left\{1+s \sqrt{1+\frac{c_{2} \lambda}{27 c_{1}^{2}}+\frac{C}{r^{4}}}\right\},
$$

with $s^{2}=1$. Thus there are two solutions obtained, only one of which can be asymptotically flat. This is one may called 'Einstein' branch. The other, $s=+1$, is the 'exotic' branch of the Boulware-Deser solution. Much of the novelty arising by these metrics is ought to its existence.

The metrics (91) appear in the literature more often in terms of the parameters $\kappa^{2}, \alpha$ and $m$ :

$$
3 ! c_{1} \equiv \kappa^{-2}, \quad \alpha \equiv \frac{c_{2}}{6 c_{1}}=\kappa^{2} c_{2}, \quad C=m \frac{8 \kappa^{2} \alpha}{3 \pi^{2}} \frac{2 \pi^{2}}{v o l_{k}} .
$$

The first relation implies also that $\kappa^{2}=8 \pi G$, in terms of the five dimensional Newton's constant. vol $_{k}$ is the volume the 3-manifold of constant curvature $k$.

Our basic formula (21) for the Hamiltonian generator reads here:

$$
\begin{aligned}
& \int_{S^{3}} c_{1} \cdot\left(\frac{1}{2} \epsilon\left(i_{\xi} \omega E^{3}\right)-\frac{1}{2} i_{\xi} \epsilon\left(\theta E^{3}\right)\right) \\
& +c_{2} \cdot\left(\epsilon\left(i_{\xi} \omega \Omega E\right)-i_{\xi} \epsilon\left(\theta\left\{\Omega_{\|}+\frac{1}{3} \theta^{2}\right\} E\right)\right),
\end{aligned}
$$

where $S^{3}$ is a large 3 -manifold of constant curvature $k$. The Killing vector $\xi$ is $\partial / \partial t$. The result is

$$
-36 \pi^{2} c_{1} g^{2} r^{2}-24 \pi^{2} c_{2} g^{2}\left(k-\frac{g^{2}}{3}\right)
$$

times $\operatorname{vol}_{k} /\left(2 \pi^{2}\right)$, where $\operatorname{vol}_{k}$ is the volume of a unit 3manifold of constant curvature $k$. This quantity diverges for large $r$.

It will be adequate to 'correct' the minimal boundary form with terms similar to those used in the five dimensional Einstein gravity in section [II]

$$
\frac{1}{2} c_{1} b_{0} \epsilon\left(E_{\|}^{4}\right)_{1}+\frac{1}{2} c_{1} b_{1} \epsilon\left(\Omega_{\|} E_{\|}^{2}\right)_{1} .
$$

The 'correction' to (94) is

$$
24 \pi^{2} c_{1} b_{0} g r^{3}+12 \pi^{2} c_{1} b_{1} k g r
$$

again times vol $_{k} /\left(2 \pi^{2}\right)$.

Define the length $l$ by

$$
-l^{-2} \equiv K=-\frac{3 c_{1}}{2 c_{2}}\left\{1+s \sqrt{1+\frac{c_{2} \lambda}{27 c_{1}^{2}}}\right\},
$$

where $K$ is the spacetime constant curvature read off from the asymptotics of the metric $g^{2}=k+r^{2} l^{-2}+\mathcal{O}\left(r^{4}\right)$. The curvature form is $\Omega^{A B}=K E^{A} E^{B}$. This is of course meaningful as long as $K$ of (97) is real.

Remark VI.1. A most important fact about the structure of the counter-terms is that they are determined solely by the asymptotic form. In other words: one needs only fix the constants $b_{0}$ and $b_{1}$ so that divergencies are removed when the metric function is exactly $g^{2}=k+r^{2} l^{-2}$.

One may consider for simplicity the $A d S$ case $K<0$, the case $K>0$ can be treated analogously at least formally. One finds that divergencies are removed for

$$
b_{0}=\frac{3}{2 l}\left(1-\frac{2 c_{2}}{9 c_{1} l^{2}}\right), \quad b_{1}=\frac{3 l}{2}\left(1+\frac{2 c_{2}}{3 c_{1} l^{2}}\right) .
$$

Then $b_{0}$ and $b_{1}$ remove the divergencies for the BoulwareDeser-Cai metric if $l$ is given by (97). $b_{1}$ is useless and not defined for $k=0 . b_{0}$ and $b_{1}$ make sense as long as $l^{-1} \neq 0$ i.e. for all cases except the asymptotically flat spacetime $s=-1$ and $\lambda=0$. On the other hand, if $k=0$ the asymptotically flat case makes sense trivially. We discuss this interesting solution separately below. The specific counterterms have also appeared in [70] but not in this form.

If we compare them to the couplings $b_{0}$ and $b_{1}$ we found in five dimensional Einstein gravity in section [II] the first terms are similar but there are $\alpha$ corrections. Interestingly the corrected couplings satisfy the exact same differential relation (59):

$$
-l^{2} \frac{d b_{0}}{d l}=\frac{d b_{1}}{d l} .
$$

That is, the relation depends on the same asymptotics to $A d S$ and (reasonably) the dimensionality but not on the theory.

Finally the Brown-York mass reads:

$$
m_{B Y}=\left(-9 \pi^{2} c_{2}+\frac{9}{2} \pi^{2} c_{1} l^{2}\right) k^{2} \frac{v_{o l}}{2 \pi^{2}}+m .
$$


For the spherically case $k=1$, discussed below, this formula agrees with the result of [41] for five dimensions derived by a set of counter-terms applying to asymptotically $A d S$ spacetimes. They were constructed in the Refs. [39 40] 42] as boundary terms respecting the conformal symmetry of $A d S$ boundary. (It was also obtained in 70 by counter-terms similar to ours, though only for the Einstein branch and not emphasized much). This is in accordance with our comments in section IID If the quasilocal mass exists i.e. the large radius is convergent, most likely the result is independent of the boundary conditions used.

Let us write down the spherical case explicitly:

$$
m_{B Y}=-9 \pi^{2} c_{2}-\frac{3 \pi}{32 G K}+m .
$$

We see the mass parameter $m$, whose setting to zero makes the metric (91) a metric of constant curvature $K$. We also see a $K$ dependent term which is exactly the same we obtained in Einstein gravity (62) in five dimensions. We also se a term which depends purely in the gravitational constant $c_{2}$. This is analogous to the vacuum energy of $A d S$ in three dimensions, $-(8 G)^{-1}$, definitely a remnant of the Chern-Simons nature of the Gauss-Bonnet term in five dimension discussed in the previous section.

This result was obtained through regularization, so it is a real type (I) case, not a type $(\mathbf{H})$ one. This holds even if we set $c_{1}=0$ decoupling the Einstein term, which kills the $K$ dependent term above. Remark III.2 does not apply and one should note the difference between this first term in (99) and Brown-York mass of Minkowski space (85) in pure Gauss-Bonnet gravity.

The flat case $k=0$ is completely different. We noted already writing down the counter-term coefficients (98), that in this case the limit $l \rightarrow \infty$ makes sense trivially. (This of course happens only for Einstein branch, $s=-1$.) I.e. the $l=\infty$ is a type $(\mathbf{H})$ case. Remark $\Pi 1.2$ may apply.

Indeed formula (99) says that for $l<\infty$, where regularization is still required, we have $m_{B Y}=m$. But if we go to the formula (94) for $l=\infty$, that $\lambda=0$ and $s=-1$, the result is convergent and equal to

$$
m_{B Y}=2 m .
$$

This is in accordance with remark III.2 extended in Lovelock gravity.

Concluding we would like to re-calculate the mass for this metric as a type (II) case, which we can always do, and obtain merely a relative value for this generator. We will define it in the usual way by: $m_{r e l}=0$ for $m=0$.

We apply formula (29) under the condition (27), using the already calculated sphere integral, formula (94). One finds

$$
\begin{aligned}
m_{r e l}= & -36 \pi^{2} c_{1}\left\{g^{2} r^{2}-g g_{\alpha} r^{2}\right\} \frac{v o l_{k}}{2 \pi^{2}} \\
& -24 \pi^{2} c_{2}\left\{g^{2}\left(k-\frac{g^{2}}{3}\right)-g g_{\alpha}\left(k-\frac{g_{\alpha}^{2}}{3}\right)\right\} \frac{v o l_{k}}{2 \pi^{2}},
\end{aligned}
$$

where $r=r_{\alpha} \rightarrow \infty$ and $m_{\alpha} \rightarrow 0$ as $\alpha \rightarrow \infty$. From that we find

$$
m_{r e l}=s^{2} m=m .
$$

The variable $m$ is the standard quantity representing the mass of the respective spacetime in this theory. We explicitly saw here that this follows from a subtraction method of calculation. There are two things that should be remarked upon.

First, the previous result holds except for $k=0$ and $\lambda=0$ of the Einstein branch $(s=-1)$ of the BoulwareDeser-Cai metric, which is a type $(\mathbf{H})$ metric as we already mentioned. In that case one finds

$$
m_{\text {rel }}=2 m .
$$

Comparing with (101) we see that they are equal. This is in accordance with the very definition of a type $(\mathbf{H})$ metric: the relative mass can be regarded as a Brown-York mass.

Secondly, there is a new thing brought up by considering relative values of generators in Lovelock gravity. All metrics are at least double-valued in this theory, thus so do the vacuum metrics. The relative mass in each branch is calculated with respect to the constant curvature spacetime (vacuum) in that branch. As the vacua are very different so is the meaning of parameter $m$ as the mass in each branch. Put in different words, the masses in each case are defined for different asymptotics and therefore their values are not comparable. For $\lambda=0$ this is all we have: the Einstein branch $(s=-1)$ is asymptotically flat.

When the cosmological constant is non-zero the BrownYork definition of the generators raises this fake degeneracy in an explicit though formal manner. The Brown-York masses of the two branches of a given Boulware-Deser metric differ by

$$
m_{B Y}^{\text {Einstein }}-m_{B Y}^{\text {exotic }}=-162 \pi^{2} \frac{c_{1}^{2}}{\lambda} \sqrt{1+\frac{c_{2} \lambda}{27 c_{1}^{2}}} .
$$

Of course this subtraction is meaningful for comparison purposes when we subtract things of the same nature. On the other hand $m_{B Y}$ is obtained by adding to the action counterterms which depend on the scale $l$. $l$ in turn, defined in (97), depends explicitly on $\lambda$ as well on the branch sign $s$, distinguishing the branches already at the level of the Lagrangian. This is like comparing masses of completely different gravitational theories. Therefore one cannot regard the Brown-York mass as a kind of absolute mass and cannot regard the $m_{B Y}$ in each branch as comparable quantities. That is, also (105) is an formal subtraction.

On the other hand if we restrict ourselves to the sector of solutions belonging to a given branch then the BrownYork mass can be regarded as a kind of absolute definition in the following sense: One calculates the mass a Hamiltonian generator of a given theory with couplings $c_{1}, c_{2}$ and $\lambda$, and no reference background or subtractions are involved. Then, inspection of the formulas (100) and (97) show that the Brown-York mass increases with $\lambda$ in the Einstein branch sector of solutions and decreases with $\lambda$ in the 
exotic branch sector. This is a reflection in the quasi-local calculation of the anti-gravity behavior of exotic branch solutions: asymptotically the exotic branch metric has an extra minus sign in the mass-dependent term.

As a physical energy of some kind, Brown-York mass is inherently related to quantum effects through the AdS /CFT correspondence [8] 9] 73]: $m_{B Y}$ equals the vacuum energy (Casimir effect) of a conformal quantum field theory living on the boundary manifold. This is a purely quantum effect coming from the zero-point energies of the field oscillators. We shall not go into boundary dual field theories here but we can point to another quantum place where the Brown-York mass makes an appearance.

In the semiclassical description of false vacuum decay (in the presence also of gravity) [74] [75] 76], one works in imaginary (Euclidean) time and considers configurations such that a bubble of real vacuum nucleates within a sea of false vacuum. The quantity which gives the rate of nucleation involves the on-shell Euclidean action. It is made of pieces of the form: (energy density) $\times$ (bubble volume), and (surface tension) $\times($ bubble area). For transitions between vacua of some curvatures $K$ one may verify that the bulk pieces of the on-shell action are proportional to

$$
m_{B Y} \cdot K^{2} \cdot(\text { bubble volume }),
$$

where $m_{B Y}$ is explicitly given by (100) for $m=0$. From the analogous formulas in Einstein gravity it is not very clear that the Brown-York mass appeared there, or better a quantity equal to it, as the result is what one expects on dimensional grounds. In Lovelock gravity there are relative coefficients that should match. We do not have a detailed explanation for this phenomenon.

\section{BOULWARE-DESER METRICS IN $6 d$}

Dimension five, being the minimum dimension for the quadratic Lovelock term to exist, exhibits certain peculiarities inexistent in dimension six or higher. This is shown for example in the qualitative differences of the black hole solutions between five and higher dimensions [61], or in the novel topological black holes presented in [77] which exist in dimension six and not in five due to a vanishing Weyl tensor. Five dimensions being minimal could be treacherous. The simple gauge theoretic construction of counter-terms we advocate could fail in dimension higher than five. This is not so. To our knowledge such a calculation has been not been presented in the literature.

The Lagrangian of the theory is easily written down, according to what we have said already in the introduction. It amounts to an insertion of one more factor of $E$ in the Lagrangian (89) (and divide $\lambda$ in the last term with 6! instead of 5 !). The field equations are obtained by varying the action functional with respect to $E$. For the standard form of the metric (90) the field equations give

$$
g-k=\frac{c_{1} r^{2}}{c_{2}}\left\{1+s \sqrt{1+\frac{c_{2} \lambda}{120 c_{1}^{2}}+\frac{C}{r^{5}}}\right\} .
$$

$C$ is an integration constant associated with the mass of the spacetime w.r.t. the asymptotic vacuum. Of course there are two branches in the solution, $s= \pm$. The relation of our couplings $c_{1}$ and $c_{2}$ to the usual couplings is: $4 ! c_{1}=\kappa^{-2}$ and $\alpha=2 ! \kappa^{2} c_{2}$, where $\kappa^{2}=8 \pi G$.

The Brown-York Hamiltonian (21), constructed out of the minimal Dirichlet boundary form, reads

$$
\begin{aligned}
& \int_{S^{4}} c_{1} \cdot\left(\frac{1}{2} \epsilon\left(i_{\xi} \omega E^{4}\right)-\frac{1}{2} i_{\xi} \epsilon\left(\theta E^{4}\right)\right) \\
& +c_{2} \cdot\left(\epsilon\left(i_{\xi} \omega \Omega E^{2}\right)-i_{\xi} \epsilon\left(\theta\left\{\Omega_{\|}+\frac{1}{3} \theta^{2}\right\} E^{2}\right)\right)
\end{aligned}
$$

where $S^{4}$ is a large 4 -manifold of constant curvature $k=$ $\pm 1,0$.

This diverges so we supplement the boundary form $\mathcal{B}_{(C)}$ with the intrinsic counter-terms

$$
\frac{1}{2} c_{1} b_{0} \epsilon\left(E_{\|}^{5}\right)_{1}+\frac{1}{2} c_{1} b_{1} \epsilon\left(\Omega_{\|} E_{\|}^{3}\right)_{1}+\frac{1}{2} c_{1} b_{2} \epsilon\left(\Omega_{\|} \Omega_{\|} E_{\|}\right)_{1} .
$$

One finds

$$
\begin{aligned}
& -256 \pi^{2} c_{1} g^{2} r^{3}-256 \pi^{2} c_{2} g^{2}\left(k-\frac{g^{2}}{3}\right) r \\
& +32 \pi^{2} c_{1} b_{0} 5 g r^{4}+32 \pi^{2} c_{1} b_{1} 3 k g r^{2}+32 \pi^{2} c_{1} b_{2} k^{2} g
\end{aligned}
$$

times $3 \operatorname{vol}_{k} /\left(8 \pi^{2}\right)$, where $\operatorname{vol}_{k}$ is the volume of the constant curvature $k$ four-manifold $S^{4}$. This additional factor is equal to one when $S^{4}$ is a sphere.

We may consider for simplicity asymptotically $A d S$ (or flat) metrics. The asymptotic $A d S$ length scale $l$ and constant curvature $K$ are defined by

$$
-l^{-2} \equiv K=-\frac{c_{1}}{c_{2}}\left\{1+s \sqrt{1+\frac{c_{2} \lambda}{120 c_{1}^{2}}}\right\}
$$

We have already mention that removing the divergencies for the asymptotic metric alone, that is when the metric is exactly given by $g^{2}=k+r^{2} l^{-2}$, suffices to remove the divergencies for all metrics (107). It is then straightforward to verify that the divergencies are removed when

$$
\begin{aligned}
& b_{0}=\frac{8}{5 l}\left(1-\frac{c_{2}}{3 c_{1} l^{2}}\right), \\
& b_{1}=\frac{4 l}{3}\left(1+\frac{c_{2}}{c_{1} l^{2}}\right), \\
& b_{2}=-l^{3}\left(1-\frac{3 c_{2}}{c_{1} l^{2}}\right) .
\end{aligned}
$$

$b_{1}$ and $b_{2}$ are useless and not defined for $k=0$.

Let now $l$ be given by (111). The Brown-York mass for the spacetime with metric (107) in the respective branch reads

$$
m_{B Y}=C \frac{c_{1}^{2}}{c_{2}} s^{2}=C \frac{c_{1}^{2}}{c_{2}}
$$


times $3 \operatorname{vol}_{k} /\left(8 \pi^{2}\right) . s= \pm$ is the branch sign. This result is in agreement with quasi-local calculations in the literature [41] 72] 69]. We derived the result from scratch in a relatively easier manner.

It is worth to mention the following. The Brown-York mass of pure $A d S$, or for that matter of pure de Sitter spacetime, given by $C=0$, is zero in six dimensions. The same we observed in four dimensions, and holds for all even dimensions [78] [41]. As a result the Brown-York of both branches of the solution is given by a similar formula.

$$
* * *
$$

From the above we reach the conclusion: If we have a Lovelock gravity in the bulk, all is required to have is a Lovelock gravity intrinsic to the boundary. Then all divergencies can be removed for a pure $A d S$ background by appropriately fixing the couplings of the boundary Lovelock theory. Then the same boundary theory applies also to asymptotically $A d S$ spacetimes. Formally, it should not be very difficult to determine the $b$ coefficients in the general Lovelock gravity, or even to more general geometric theories. A better understanding of the whole thing would be much better and we shall leave such an analysis for a separate work.

\section{FLAT FROM $A D S$}

The quasi-local charges $H_{\xi}(\alpha)$, relation (23), were introduced as candidates of the total content of a spacelike section $\Sigma^{\alpha}$ bounded by $S^{\alpha}$ in the respective conserved charge. Such a quantity should possess a number of properties, discusses in the works cited in section [II Of Of them we prove none apart from the naturalness of their definition i.e. them being essentially on-shell values of the HamiltonJacobi theory generators. On this basis at least, one may study them and realize that one can extract interesting information from them. For example they operate as interpolations between the singular asymptotical flat case and the non-zero cosmological constant metrics.

As a first example consider $A d S$ metric in three dimension, discussed in section III]. The mass was calculated there as the large $r$ limit of the quantity $-2 \pi c_{1} g^{2}-$ $2 \pi c_{1} b_{0} g r$. After a little algebra one may write the result in the form

$$
m_{B Y}(r)=-\frac{1}{4 G}\left(1+\frac{1}{\sqrt{1+(l / r)^{2}}}\right)^{-1} .
$$

One first observes that this is an increasing function of $r$ : it is larger the larger is the size of the region in space. Specifically goes from the value $-(4 G)^{-1}$ obtained in the 'small sphere' limit $r \rightarrow 0$, to the value $-(8 G)^{-1}$ in the limit $r \rightarrow \infty$. The latter value is of course the value of the Brown-York mass (47) of $A d S$ spacetime in dimension three.

On the other hand, the 'small sphere' result $-(4 G)^{-1}$ can be recognized as the Minkowski spacetime Brown-York mass (78) we found in section IV. The reason is simple: The result depends on the lengths $l$ and $r$ only through the dimensionless ratio $l / r$. Therefore $r \rightarrow 0$ is equivalent to $l \rightarrow \infty$. This is nothing but taking the asymptotically flat limit before letting the size of the region to go to infinity. Letting $r$ become large is then trivial in this example and one obtains from the $A d S$ space quasilocal charge (114) the flat space Brown-York mass.

Thus there is actually continuity in the limit $l \rightarrow \infty$. (This is also one more reason why we should apparently take a non-zero Brown-York defined mass for the Minkowski spacetime seriously). The reason why it is possible obtain the flat space result for the $A d S$ space one, is that the former is a type $(\mathbf{H})$ case. One may verify that everything said above applies also to the five-dimensional $k=0$ Boulware-Deser-Cai metric of Lovelock gravity.

We may now extend remark $\amalg I .2$ which concerns the types $(\mathbf{H})$ cases:

Remark VIII.1. The quasi-local values of the Hamiltonian generators in type $(\mathbf{H})$ cases can be obtained as the 'small sphere' limit of the quasi-local generators of the associated $A d S$ metrics.

The need for the extension is that in the limit $l \rightarrow \infty$ the mass not always changes by a factor of 2 . This can be seen by comparing the mass (100) of the Boulware-Deser metric and the mass (85) of the Minkowski spacetime in pure Gauss-Bonnet gravity (i.e. no Einstein term is present) which is a type $(\mathbf{H})$ case.

To make things completely explicit let's consider pure Gauss-Bonnet gravity in the presence of a cosmological constant. This is the theory (89) for $c_{1}=0$. Everything we need can be obtained from the full Lovelock gravity results of the previous section, as neither the length $l$ nor the counter-term coefficients $c_{1} b_{0}$ and $c_{1} b_{1}$ are singular in setting $c_{1}=0$. In any case it is straightforward to verify that the obtained counter-terms work. One finally finds

$$
\begin{aligned}
& m_{B Y}(r)=4 \pi^{2} c_{2} \times \\
& \times\left\{\left(3 \frac{r^{2}}{l^{2}}-2 \frac{r^{4}}{l^{4}}\right)\left(\sqrt{1+\frac{l^{2}}{r^{2}}}-1\right)+\frac{r^{2}}{l^{2}}-4\right\} .
\end{aligned}
$$

In the limit $r \rightarrow \infty$ we obtain $-9 \pi^{2} c_{2}$. This is the first term of the full formula (100) as expected. In the small sphere limit $r \rightarrow 0$ one obtains $-16 \pi^{2} c_{2}$ which indeed is the $l=\infty$ result (85).

Now, if $m_{B Y}(r)$ is the amount of total energy contained in a sphere of radius $r$ in these examples, why does it not vanish in the small sphere limit?

First of all one can always set a constant value of energy to zero by suitably fixing the constant $H^{0}$ in (21) and (23). Consider then the example of $A d S_{3}$. If we fix $H^{0}$ such that $m_{B Y}(r)$ vanishes in the small sphere limit then the Brown-York mass in spacetime is $-(4 G)^{-1}$, instead of $-(8 G)^{-1}$. But it is not that easy to change our minds and fiddle the latter value; it can be re-discovered as the mass of a state in the boundary conformal field theory which 
can be identified with $A d S_{3}$ [79]. It is then advisable to relax the interpretation of the quasi-local mass as the mass 'contained' in a spatial region. A better use of it has been pointed out in this section.

\section{SUMMARY AND COMMENTS}

Covariant phase space methods are used in the first order formulation of Einstein and Lovelock gravity for the derivation of the Hamiltonian generators. Many known results and some new ones are derived and discussed in this context. Relations between them and phenomena which arise as we go from three to six dimensions are studied. We find that in the odd dimensions, if the higher possible Lovelock term is included, one should attribute a Brown-York energy in Minkowski spacetime. This is intimately related to the effect of the latter to create deficit angles singularities in space. The relation of the flat spacetime results to those of the associated $A d S$ spaces is discussed. In certain cases, where a minimal boundary Lagrangian is adequate for convergence, the flat space result derives from the small sphere limit of the $A d S$ one. In higher dimensions, five and six, with Lovelock gravity turned on, the simplicity of the first order formulation of the theory (in differential forms notation) with Dirichlet boundary conditions and its agreement with other methods is emphasized. This agreement is consistent with the analysis of the first sections of the paper which suggests that all convergent quasi-local definitions should agree up to a phase space constant. The counter-term coefficients exhibit a structure: for example, in five dimensions those coefficients of the full Lovelock gravity obey the same differential relation with those of Einstein; something similar can be done in dimension six. It becomes rather clear that a boundary Lovelock gravity can regularize the divergencies of a Lovelock gravity in the bulk. Study of this general problem is left for future work.

Well defined Hamiltonian generators rest on the existence of a non-degenerate symplectic form, which we tacitly assumed. This is not guarantied in Lovelock gravity. In an analogous particle system with Lagrangian $L(x, v)$ the symplectic form would read $d(d L / d v) \wedge d x$. This gives trouble if $L$ has an inflection point. At such points accelaration terms drop out the field equations. Neither such a system nor Lovelock gravity have an priori well posed initial value problem [14]. We may derive a Hamiltonian in the space of solutions only away from those points [80].

An interesting way out of this problem, as well as out of worst problems of causal evolution arising for piecewise smooth solutions [81], was put forward in Ref. [82]. There is a class of Lovelock gravities, which are not too special i.e. they are not of measure zero in coupling space, whose actions are interpreted as a Weyl tube volume (see [82] and references therein). The idea is that as long as the Weyl tube does not intersect itself, in which case a Lovelock action does represent the tube volume, the associated gravity is safe. To the extent this is correct one could consistently do Hamiltonian theory in those theories.

\section{Acknowledgments}

We thank S. Willison for helpful discussions. Part of this work was done during a pleasant visit to CECS (Centro de Estudios Científicos); the Center is thanked for hospitality.

\section{Appendix}

\section{Appendix A: Second fundamental form}

Consider a non-null hypersurface embedded in spacetime with a unit vector field $\zeta^{A}$ normal to it. $\zeta \cdot \zeta= \pm 1$. We denote by $i_{\|}^{*}$ the pull-back into the hypersurface. Let $E_{\|}^{a}$ be an intrinsic vielbein and $\omega_{\|}^{a b}$ be an intrinsic connection. Lower case Lorentz indices label tensor components normal to $\zeta$.

Impose a coincidence condition: $i_{\|}^{*} \omega^{a b}=\omega_{\|}^{a b}$ and $i_{\|}^{*} E^{a}=$ $E_{\|}^{a}$ i.e. the induced fields coincide with the intrinsic fields. This in accordance with section 【A This is possible in each smooth component of the boundary.

Let us impose the conditions that the induced fields held fixed:

$$
i_{\|}^{*} \delta \omega^{a b}=0 \quad \text { and } \quad i_{\|}^{*} \delta E^{a}=0 .
$$

Then also $\delta E_{\|}^{a}=0$ and $\delta \omega_{\|}^{a b}=0$, by the coincidence condition. In words: the induced fields are not varied under Euler-Lagrange variations; respecting the coincidence condition the intrinsic fields are held fixed to their values. This is the 'Dirichlet boundary conditions' in first order formalism.

We now define

$$
\boldsymbol{\theta}:=i_{\|}^{*}\left(\omega-\omega_{\|}\right) .
$$

This is the second fundamental form of the embedding of the hypersurface into spacetime. Clearly, the non-zero components of $\boldsymbol{\theta}$ have one index in the normal direction of the hypersurface.

The fields $\omega_{\|}$and $E_{\|}$can be regarded as a bulk fields which agree with the intrinsic fields when pulled back into the hypersurface. We often use the quantity $\theta \equiv \omega-\omega_{\|}$. Of course $\boldsymbol{\theta}=i_{\|}^{*} \theta$.

\section{Appendix B: boundary forms}

Consider for simplicity Einstein gravity in three dimensions. The Lagrangian is $\mathcal{L}=\frac{1}{2} c_{1} \epsilon(\Omega E)$.

We have

$$
\delta \epsilon(\Omega E)=d \epsilon(\delta \omega E)+\delta \Psi \cdot \mathcal{E} .
$$

We have

$$
\begin{aligned}
i_{\|}^{*} \epsilon(\delta \omega E) & =i_{\|}^{*} \epsilon\left(\delta\left[\omega-\omega_{\|}\right] E\right)+i_{\|}^{*} \epsilon\left(\delta \omega_{\|} E\right) \\
& =\delta\left\{i_{\|}^{*} \epsilon\left(\left[\omega-\omega_{\|}\right] E\right)\right\}+i_{\|}^{*} \delta \Psi \cdot \mathcal{E}_{b d y},
\end{aligned}
$$


where $\mathcal{E}_{b d y}$ are the boundary equations of motion, essentially the quasi-local energy momentum and spin tensors $Q_{\text {quasi-local }}$ of section II A. By (A.1) this term vanishes.

Thus we have that under Dirichlet conditions

$$
\delta_{(C)}\left\{\int_{\mathcal{R}} \epsilon(\Omega E)-\int_{\partial \mathcal{R}} \epsilon\left(\left[\omega-\omega_{\|}\right] E\right)\right\}=\int_{\mathcal{R}} \delta_{(C)} \Psi \cdot \mathcal{E} .
$$

The symbol $\delta_{(C)}$, which we use in the main text, emphasizes that the variations are done under the specific boundary conditions. Therefore the action

$$
S=\frac{1}{2} c_{1} \int_{\mathcal{R}} \epsilon(\Omega E)-\frac{1}{2} c_{1} \int_{\partial \mathcal{R}} \epsilon(\theta E),
$$

has an extremum on-shell under $\delta_{(C)}$. This is Einstein-
Hilbert action with a Gibbons-Hawking term. The boundary form $\mathcal{B}_{(C)}$ used in the next is read off from it. The generalization of this action in higher dimensions amounts to simply inserting an equal number of factors of $E$ in the bulk and boundary form, as one may verify.

In Einstein-Gauss-Bonnet gravity, we mostly use in this paper, the boundary form was written down in Ref. 58], and for Lovelock gravity in Ref. [83]. This can be constructed in all Lovelock gravities via a Chern-Weil procedure which provides the transgression forms in the associated topological problems, see e.g. [16]. In that spirit they where constructed in [84]. A general method for construction of the boundary forms for general geometric Lagrangians was presented in [85].
[1] H. Bondi, M. G. J. van der Burg, and A. W. K. Metzner, Proc. Roy. Soc. Lond. A269, 21 (1962).

[2] R. K. Sachs, Proc. Roy. Soc. Lond. A270, 103 (1962).

[3] R. L. Arnowitt, S. Deser, and C. W. Misner, Phys. Rev. 116, 1322 (1959).

[4] L. F. Abbott and S. Deser, Nucl. Phys. B195, 76 (1982).

[5] J. D. Brown and J. York, James W., Phys. Rev. D47, 1407 (1993), arXiv:gr-qc/9209012.

[6] V. Balasubramanian and P. Kraus, Commun. Math. Phys. 208, 413 (1999), arXiv:hep-th/9902121.

[7] V. Balasubramanian, J. de Boer, and D. Minic, Phys. Rev. D65, 123508 (2002), arXiv:hep-th/0110108.

[8] J. M. Maldacena, Adv. Theor. Math. Phys. 2, 231 (1998), arXiv:hep-th/9711200.

[9] E. Witten, Adv. Theor. Math. Phys. 2, 253 (1998), arXiv:hep-th/9802150.

[10] G. 't Hooft, (1993), arXiv:gr-qc/9310026.

[11] L. Susskind, J. Math. Phys. 36, 6377 (1995), arXiv:hepth/9409089.

[12] J. D. Brown, S. R. Lau, and J. York, James W., (2000), arXiv:gr-qc/0010024.

[13] D. Lovelock, J. Math. Phys. 12, 498 (1971).

[14] Y. Choquet-Bruhat, J. Math. Phys. 29, 1891 (1988).

[15] B. Zumino, Phys. Rept. 137, 109 (1986).

[16] T. Eguchi, P. B. Gilkey, and A. J. Hanson, Phys. Rept. 66, 213 (1980).

[17] S. I. Goldberg, Curvature and Homology (Dover, 1998).

[18] C. Crnkovic and E. Witten, Print-86-1309 (PRINCETON).

[19] V. I. Arnold, Mathematical Methods of Classical Mechanics (Springer-Verlag, 1986).

[20] R. Abraham and J. R. Marsden, Foundations of Mechanics (Addison-Wesley, 1987).

[21] M. Henningson and K. Skenderis, JHEP 07, 023 (1998), arXiv:hep-th/9806087.

[22] Y. S. Myung, Mod. Phys. Lett. A16, 2353 (2001), arXiv:hep-th/0110123.

[23] A. M. Ghezelbash and R. B. Mann, JHEP 01, 005 (2002), arXiv:hep-th/0111217.

[24] S. R. Lau, Phys. Rev. D60, 104034 (1999), arXiv:grqc/9903038.

[25] R. B. Mann, Phys. Rev. D61, 084013 (2000), arXiv:hepth/9904148.

[26] R. B. Mann, Phys. Rev. D60, 104047 (1999), arXiv:hep- th/9903229.

[27] P. Kraus, F. Larsen, and R. Siebelink, Nucl. Phys. B563, 259 (1999), arXiv:hep-th/9906127.

[28] J. D. Brown, S. R. Lau, and J. York, James W., Phys. Rev. D59, 064028 (1999), arXiv:gr-qc/9810003.

[29] J. Lee and R. M. Wald, J. Math. Phys. 31, 725 (1990).

[30] R. M. Wald, Phys. Rev. D48, 3427 (1993), arXiv:grqc/9307038.

[31] V. Iyer and R. M. Wald, Phys. Rev. D50, 846 (1994), arXiv:gr-qc/9403028.

[32] V. Iyer and R. M. Wald, Phys. Rev. D52, 4430 (1995), arXiv:gr-qc/9503052.

[33] T. Regge and C. Teitelboim, Ann. Phys. 88, 286 (1974).

[34] R. Benguria, P. Cordero, and C. Teitelboim, Nucl. Phys. B122, 61 (1977).

[35] S. A. Hayward, Phys. Rev. D49, 831 (1994), arXiv:grqc/9303030.

[36] C. W. Misner and D. H. Sharp, Phys. Rev. 136 (1964).

[37] S. A. Hayward, Phys. Rev. D53, 1938 (1996), arXiv:grqc/9408002.

[38] H. Maeda and M. Nozawa, Phys. Rev. D77, 064031 (2008), arXiv:0709.1199.

[39] P. Mora, R. Olea, R. Troncoso, and J. Zanelli, JHEP 06, 036 (2004), arXiv:hep-th/0405267.

[40] P. Mora, R. Olea, R. Troncoso, and J. Zanelli, (2004), arXiv:hep-th/0412046.

[41] G. Kofinas and R. Olea, Phys. Rev. D74, 084035 (2006), arXiv:hep-th/0606253.

[42] R. Olea, JHEP 04, 073 (2007), arXiv:hep-th/0610230.

[43] O. Miskovic and R. Olea, JHEP 10, 028 (2007), arXiv:0706.4460.

[44] G. Kofinas and R. Olea, JHEP 11, 069 (2007), arXiv:0708.0782.

[45] K. Skenderis, Class. Quant. Grav. 19, 5849 (2002), arXiv:hep-th/0209067.

[46] S. W. Hawking and G. T. Horowitz, Class. Quant. Grav. 13, 1487 (1996), arXiv:gr-qc/9501014.

[47] A. Ashtekar, J. Engle, and D. Sloan, Class. Quant. Grav. 25, 095020 (2008), arXiv:0802.2527.

[48] R. B. Mann, Class. Quant. Grav. 14, L109 (1997), arXiv:grqc/9607071.

[49] D. R. Brill, J. Louko, and P. Peldan, Phys. Rev. D56, 3600 (1997), arXiv:gr-qc/9705012. 
[50] L. Vanzo, Phys. Rev. D56, 6475 (1997), arXiv:grqc/9705004.

[51] G. W. Gibbons, M. J. Perry, and C. N. Pope, Phys. Rev. Lett. 95, 231601 (2005), arXiv:hep-th/0507034.

[52] S. Deser, R. Jackiw, and G. 't Hooft, Ann. Phys. 152, 220 (1984).

[53] A. Ashtekar and M. Varadarajan, Phys. Rev. D50, 4944 (1994), arXiv:gr-qc/9406040.

[54] G. Clement, Int. J. Theor. Phys. 24, 267 (1985).

[55] D. Marolf and L. Patino, Phys. Rev. D74, 024009 (2006), arXiv:hep-th/0604127.

[56] E. Gravanis and S. Willison, Phys. Rev. D75, 084025 (2007), arXiv:gr-qc/0701152.

[57] R. Jackiw, Lectures given at SILARG VII, Cocoyoc, Mexico, Dec 1990.

[58] R. C. Myers, Phys. Rev. D36, 392 (1987).

[59] D. G. Boulware and S. Deser, Phys. Rev. Lett. 55, 2656 (1985).

[60] J. T. Wheeler, Nucl. Phys. B268, 737 (1986).

[61] R.-G. Cai, Phys. Rev. D65, 084014 (2002), arXiv:hepth/0109133.

[62] S. Deser and B. Tekin, Phys. Rev. Lett. 89, 101101 (2002), arXiv:hep-th/0205318.

[63] S. Deser and B. Tekin, Phys. Rev. D67, 084009 (2003), arXiv:hep-th/0212292.

[64] S. Deser and B. Tekin, Phys. Rev. D75, 084032 (2007), arXiv:gr-qc/0701140.

[65] C. Charmousis and A. Padilla, JHEP 12, 038 (2008), arXiv:0807.2864.

[66] M. Cvetic, S. Nojiri, and S. D. Odintsov, Nucl. Phys. B628, 295 (2002), arXiv:hep-th/0112045.

[67] A. Padilla, Class. Quant. Grav. 20, 3129 (2003), arXiv:grqc/0303082

[68] M. H. Dehghani, N. Bostani, and A. Sheikhi, Phys. Rev.
D73, 104013 (2006), arXiv:hep-th/0603058.

[69] J. T. Liu and W. A. Sabra, (2008), arXiv:0807.1256.

[70] Y. Brihaye and E. Radu, Phys. Lett. B661, 167 (2008), arXiv:0801.1021.

[71] Y. Brihaye and E. Radu, JHEP 09, 006 (2008), arXiv:0806.1396.

[72] Y. Brihaye and E. Radu, Phys. Lett. B678, 204 (2009), arXiv:0812.3296.

[73] S. S. Gubser, I. R. Klebanov, and A. M. Polyakov, Phys. Lett. B428, 105 (1998), arXiv:hep-th/9802109.

[74] S. R. Coleman, Phys. Rev. D15, 2929 (1977).

[75] J. Callan, Curtis G. and S. R. Coleman, Phys. Rev. D16, 1762 (1977).

[76] S. R. Coleman and F. De Luccia, Phys. Rev. D21, 3305 (1980).

[77] C. Bogdanos, C. Charmousis, B. Gouteraux, and R. Zegers, JHEP 10, 037 (2009), arXiv:0906.4953.

[78] R. Aros, M. Contreras, R. Olea, R. Troncoso, and J. Zanelli, Phys. Rev. D62, 044002 (2000), arXiv:hep-th/9912045.

[79] A. Strominger, JHEP 02, 009 (1998), arXiv:hepth/9712251.

[80] C. Teitelboim and J. Zanelli, Class. Quant. Grav. 4, 125 (1987).

[81] C. Garraffo, G. Giribet, E. Gravanis, and S. Willison, J. Math. Phys. 49, 042502 (2008), arXiv:0711.2992.

[82] S. Willison, Phys. Rev. D80, 064018 (2009), arXiv:0904.3224.

[83] F. Mueller-Hoissen, Nucl. Phys. B337, 709 (1990).

[84] E. Gravanis and S. Willison, J. Math. Phys. 45, 4223 (2004), arXiv:hep-th/0306220.

[85] E. Gravanis and S. Willison, J. Math. Phys. 50, 122505 (2009), arXiv:0901.1079. 